Reassignment of the $\mathrm{O}_{2}$ spectrum just below dissociation threshold based on ab initio calculations

Mirjam C. G. N. van Vroonhoven and Gerrit C. Groenenboom

Citation: The Journal of Chemical Physics 117, 5240 (2002); doi: 10.1063/1.1499493

View online: $\mathrm{http} / / / \mathrm{dx}$.doi.org/10.1063/1.1499493

View Table of Contents: http://aip.scitation.org/toc/jcp/117/11

Published by the American Institute of Physics

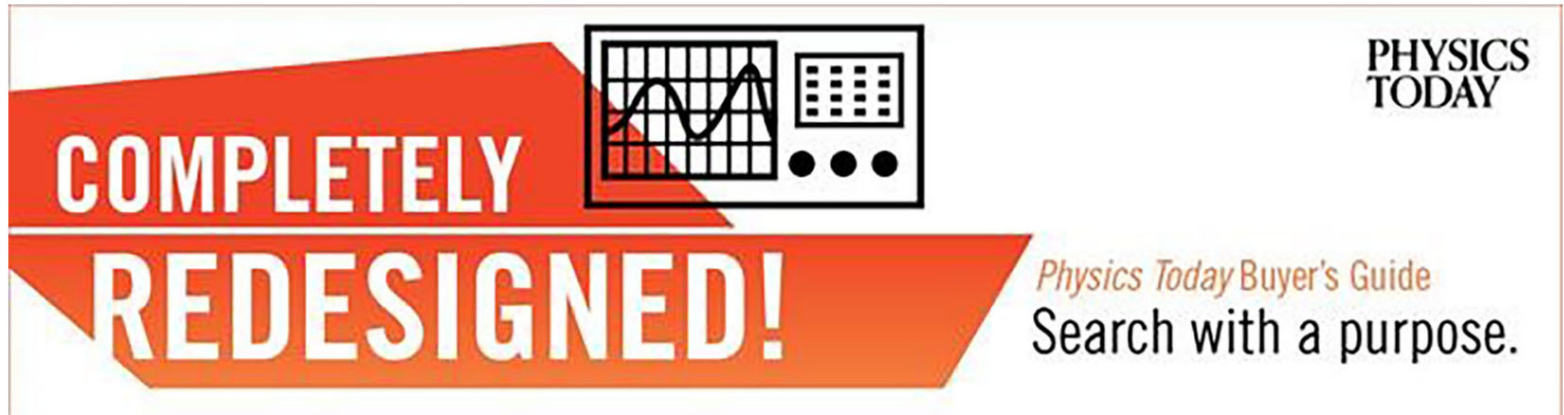




\title{
Reassignment of the $\mathrm{O}_{2}$ spectrum just below dissociation threshold based on ab initio calculations
}

\author{
Mirjam C. G. N. van Vroonhoven ${ }^{\text {a) }}$ and Gerrit C. Groenenboom ${ }^{\text {b) }}$ \\ Institute of Theoretical Chemistry, University of Nijmegen, Toernooiveld 1, 6525 ED Nijmegen, \\ The Netherlands
}

(Received 17 May 2002; accepted 19 June 2002)

\begin{abstract}
Vibrational Herzberg bands of the $\mathrm{O}_{2}$ molecule just below its first $\mathrm{O}\left({ }^{3} P\right)+\mathrm{O}\left({ }^{3} P\right)$ dissociation limit are since long-known to be perturbed. Jenouvrier et al. [J. Mol. Spectrosc. 198, 136 (1999)] assigned the cause of the perturbations to five vibrational levels supported by the shallow minimum in the $1{ }^{3} \Pi_{u}$ potential energy curve around $5.5 a_{0}$. Using ab initio potential energy curves and spin-orbit couplings from previous work [J. Chem. Phys. 116, 1954 (2002)] we present a full quantum calculation of all ungerade rotation-vibration-electronic states of oxygen just below the dissociation threshold, through a total angular momentum quantum number of $J=19$. This calculation shows that the original assignment, based on a Hund's case (a) model of a regular $1{ }^{3} \Pi_{u}$ multiplet was not correct. Based on our calculation we present a new assignment of the perturbing states: $1{ }^{3} \Pi_{u, \Omega=2}(v=0), 1{ }^{3} \Pi_{u, 1}(0), 1{ }^{3} \Pi_{u, 2}(1), 1{ }^{3} \Pi_{u, 1}(1)$, and $1{ }^{3} \Pi_{u, 0^{-}}(0)$ in order of ascending term values. We show the new assignment to be consistent with experimental data and we also propose new spectroscopic parameters for the perturbing states. (C) 2002 American Institute of Physics. [DOI: 10.1063/1.1499493]
\end{abstract}

\section{INTRODUCTION}

Eight ungerade states of $\mathrm{O}_{2}$ correlate with its lowest dissociation limit $\mathrm{O}\left({ }^{3} P\right)+\mathrm{O}\left({ }^{3} P\right)$. Three of these states are very well characterized by extensive spectroscopic studies ${ }^{1-11}$ of the so-called Herzberg bands, bands corresponding to transitions from the $X^{3} \Sigma_{g}^{-}$ground state to the $A^{3} \Sigma_{u}^{+}$(Herzberg I), $c^{1} \Sigma_{u}^{-}$(Herzberg II), and $A^{\prime 3} \Delta_{u}$ (Herzberg III) states. The other five ungerade states, $1{ }^{3} \Pi_{u}, 1{ }^{5} \Sigma_{u}^{-}, 1{ }^{5} \Pi_{u}, 1{ }^{1} \Pi_{u}$, and $2^{3} \Sigma_{u}^{+}$, are difficult to detect because these potentials have only shallow minim at large internuclear separations $\left(r>5 a_{0}\right)$, which leads to very unfavorable Franck-Condon overlap with the ground state that has an $r_{0}=2.29 a_{0}$. Still, the spin-orbit interaction between all these states affects the $\mathrm{O}\left({ }^{3} P_{j}\right)$ fine-structure branching ratios for photodissociation of $\mathrm{O}_{2}$ in the Herzberg continuum. ${ }^{12,13}$ These interactions (together with spin-orbit interactions among the gerade states) are also responsible for the excitation and quenching of the fine-structure levels in collisions among oxygen atoms. ${ }^{14,15} \mathrm{~A}$ quantitative understanding of processes affecting finestructure level populations is very important in atmospheric chemistry. ${ }^{16}$ Clearly $a b$ initio calculations are a valuable source of information about these potentials and couplings. However, the open shell character of these states complicates the proper treatment of the electron correlation and the basis set superposition error. ${ }^{12}$ Thus, spectroscopic information on states in the so-called recoupling region $\left(r \approx 4-7 a_{0}\right)$ can provide useful benchmark information.

A glimpse of the spectroscopy of the weakly bound states is provided by perturbations in the Herzberg bands that

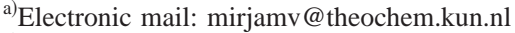

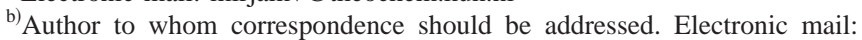
gerritg@theochem.kun.nl
}

occur less than about $110 \mathrm{~cm}^{-1}$ below the dissociation limit. The weakly bound states cause a characteristic pattern of deviations from straight lines which emerge when the term values of the observed Herzberg levels are plotted as a function of $J(J+1)$. Perturbations in the $v=11$ band of the $A^{3} \Sigma_{u}^{+}$state were first noted by Herzberg ${ }^{2}$ in 1952. In 1986 Borrell et al. ${ }^{5}$ report perturbations in the $N=9,11$, and 13 rotational levels of this band. They suggest, based on potentials of Saxon and Liu, ${ }^{17}$ that the ${ }^{5} \Sigma_{u}^{-}$state is the perturber. In 1991, Partridge et al. ${ }^{18}$ perform more advanced ab initio calculations on these states and propose the $1{ }^{3} \Pi_{u}$ state, which has a deeper well, as the more likely candidate.

Jenouvrier et al. ${ }^{19}$ recently remeasured the Herzberg bands with high resolution Fourier transform spectroscopy, identifying perturbations in the $A^{3} \Sigma_{u}^{+}(v=11)$, $c^{1} \Sigma_{u}^{-}(v=18,19)$, and $A^{\prime}{ }^{3} \Delta_{u, 2}(v=12)$ Herzberg bands. They attributed the perturbations to five $1^{3} \Pi_{u}$ levels. Assuming that this state is a regular Hund's case (a) multiplet, the perturbing levels were assigned to be $1^{3} \Pi_{u, \Omega=1}(v=0)$, $1{ }^{3} \Pi_{u, 2}(0), 1{ }^{3} \Pi_{u, 0}(1), 1{ }^{3} \Pi_{u, 1}(1)$, and $1{ }^{3} \Pi_{u, 2}(1)$ in order of ascending term values. It was assumed that the $1^{3} \Pi_{u, 0}(0)$ level was the lowest $1{ }^{3} \Pi_{u}$ level, although no corresponding perturbations were observed. In a previous paper, ${ }^{12}$ which we will refer to as Paper I, we calculated potential energy curves for all the electronic states involved at the internally contracted multireference configuration interaction level plus Pople size consistency correction, employing large basis sets. We also computed all diagonal and off-diagonal spin-orbit couplings amongst those states at the complete active space self consistent field level. We employed these ab initio data in a semiclassical study of the photodissociation of $\mathrm{O}_{2}$ in the Herzberg continuum. ${ }^{13}$

In the present paper we use the ab initio results in a full 
quantum calculation of the spectroscopically observed levels of $\mathrm{O}_{2}$ just below dissociation. Our calculations indicate that the major perturbing state, $1^{3} \Pi_{u}$, cannot be described by a regular Hund's case (a) state in the relevant region of $r \approx 5.5$ $a_{0}$. In fact, the diagonal spin-orbit coupling matrix element is negative and spin-orbit couplings with other electronic states cannot be neglected. Although the electronic states are mixed we can assign the perturbing state and propose a new assignment by comparing the exact results with more approximate Hund's case (a) and (c) calculations. Many of the observed perturbations involve rather high rotational levels with $J$ up to 17. Since in some cases the experimental $J=0$ spectroscopic parameters depend on the assignment and the too simplistic Hund's case (a) model, we also performed calculations for the rotational levels that were actually observed. The pattern of perturbations depends very sensitively on the position of the $1^{3} \Pi_{u}(0,1)$ levels relative to the highly vibrationally excited Herzberg levels. To achieve better agreement with experiment we slightly scaled and adjusted our potentials. This scaling also allows us to draw a conclusion about the accuracy of the ab initio calculations of potential energy curves of and couplings between weakly interacting open shell atoms. We also use the plot of the term values versus $J(J+1)$ to extract the rotational constant of the "pure" $1{ }^{3} \Pi_{u}$ level via a fit of the term values to a polynomial in $J(J+1)$. Since most $1{ }^{3} \Pi_{u}$ levels are mixed with the Herzberg states, computation of the rotational constant as the expectation value of $1 / 2 \mu r^{2}$ typically yields larger rotational constants.

The outline of this paper is as follows: In Sec. II we discuss the theoretical aspects of our calculation, the different parts of the Hamiltonian, and the basis functions used to expand the wave functions for the rotation-vibrationelectronic (RVE) states. We also give some computational details on the discrete variable representation used for the radial nuclear motion. In Sec. III, we discuss our potentials, the rotationless vibrational level positions in the Hund's case (a) and (c) approximations, and in a full coupled calculation, the scaling and adjusting of the potential energy curves, and finally in Sec. III F the results including the full rotational part of the Hamiltonian. We give our conclusions in Sec. IV. In the Appendix we define our basis functions, and derive their behavior under the parity operation. We also present a new derivation of the rotational kinetic energy matrix element which avoids the use of Hougen's isomorphic Hamiltonian. ${ }^{20}$

\section{THEORY}

We compute RVE bound states of the $\mathrm{O}_{2}$ molecule as eigenstates of the total Hamiltonian,

$$
\hat{H}=\hat{H}_{\mathrm{Coul}}+\hat{H}_{\mathrm{SO}}+\hat{H}_{\mathrm{vib}}+\hat{H}_{\mathrm{rot}},
$$

where $\hat{H}_{\text {Coul }}$ is the usual Coulombic Hamiltonian in the clamped nuclei approximation and $\hat{H}_{\text {SO }}$ is the Breit-Pauli spin-orbit (SO) Hamiltonian. The nuclear radial kinetic energy is given by $\hat{H}_{\text {vib }}=-\left(\hbar^{2} / 2 \mu\right) r^{-1}\left(\partial^{2} / \partial r^{2}\right) r$, where $r$ is the interatomic distance and $\mu=7.9975 u$ is the reduced mass of ${ }^{16} \mathrm{O}^{16} \mathrm{O}$. The rotational energy part $\hat{H}_{\text {rot }}$ will be discussed below. The electronic adiabatic Born-Oppenheimer (ABO) wave functions, i.e., the eigenfunctions of $\hat{H}_{\text {Coul }}$, are taken from paper I. The ABO states are pure Hund's case (a) wave functions and we denote them by $|(L) \Lambda S \Sigma ; r\rangle$, where $\Lambda$ and $\Sigma$ are the projections, respectively, of the total electronic angular momentum $(\underline{\hat{L}})$ and the electron spin $(\underline{\hat{S}})$ on the internuclear axis. At large internuclear separation $L$ is also a good quantum number and we use it to distinguish between the triplet states $A^{3} \Sigma_{u}^{+}(L=0)$ and $2^{3} \Sigma_{u}^{+}(L=2)$ of the same $\left(D_{\infty h}\right)$ symmetry, as was explained in Paper I.

In that paper we presented analytic fits to the ABO potentials $V_{L|\Lambda| S}(r)$ for different values of $L,|\Lambda|$, and $S$, which are defined by

$$
V_{L|\Lambda| S}(r)=\left\langle(L) \Lambda S \Sigma ; r\left|\hat{H}_{\text {Coul }}\right|(L) \Lambda S \Sigma ; r\right\rangle .
$$

In addition, we computed $r$-dependent $\mathrm{SO}$ coupling matrix elements which, using the Wigner-Eckart theorem may be expressed as

$$
\begin{aligned}
\left\langle(L) \Lambda S \Sigma ; r\left|\hat{H}_{\mathrm{SO}}\right|\left(L^{\prime}\right) \Lambda^{\prime} S^{\prime} \Sigma^{\prime} ; r\right\rangle & \\
= & (-1)^{S-\Sigma}\left(\begin{array}{ccc}
S & 1 & S^{\prime} \\
-\Sigma & \left(\Sigma-\Sigma^{\prime}\right) & \Sigma^{\prime}
\end{array}\right) \\
& \times\left\langle(L) \Lambda S ; r\left\|\hat{H}_{\mathrm{SO}}(r)\right\|\left(L^{\prime}\right) \Lambda^{\prime} S^{\prime} ; r\right\rangle,
\end{aligned}
$$

where the quantity between large parentheses is a $3 j$ symbol. Note that matrix elements are only nonzero when $\Delta \Omega=0$, where $\Omega=\Lambda+\Sigma$. We provided fits to the 21 independent reduced SO matrix elements $\left\langle(L) \Lambda S ; r\left\|\hat{H}_{\mathrm{SO}}(r)\right\|\left(L^{\prime}\right) \Lambda^{\prime} S^{\prime} ; r\right\rangle$. We also presented in Paper I the only nonvanishing radial derivative coupling matrix element amongst the eight $\mathrm{ABO}$ states, i.e., $\left\langle A^{3} \Sigma_{u}^{+} ; r|\partial / \partial r| 2{ }^{3} \Sigma_{u}^{+} ; r\right\rangle$. However, in semiclassical calculations on the photodissociation of $\mathrm{O}_{2}$ we found that the effect of this coupling just above the dissociation limit is negligible and hence we do not include it in the present bound state calculations.

\section{A. The rotational Hamiltonian}

The rotational Hamiltonian is given by

$$
\begin{aligned}
\hat{H}_{\mathrm{rot}}^{\text {(exact) }}= & \frac{1}{2 \mu r^{2}}\left[\left(\hat{J}^{2}-\hat{J}_{z}^{2}\right)+\left(\hat{L}^{2}-\hat{L}_{z}^{2}\right)+\left(\hat{S}^{2}-\hat{S}_{z}^{2}\right)\right. \\
& +\left(\hat{L}^{+} \hat{S}^{-}+\hat{L}^{-} \hat{S}^{+}\right)-\left(\hat{L}^{+} \hat{J}^{-}+\hat{L}^{-} \hat{J}^{+}\right) \\
& \left.-\left(\hat{S}^{+} \hat{J}^{-}+\hat{S}^{-} \hat{J}^{+}\right)\right],
\end{aligned}
$$

in body-fixed operators, with $\underline{\hat{J}}=\underline{\hat{l}}+\underline{\hat{L}}+\underline{\hat{S}}$ and $\underline{\underline{l}}$ is the angular momentum associated with the rotation of the nuclei. In the Appendix we derive this Hamiltonian and its matrix elements. Asymptotically, the states we are considering are derived from coupling atomic $P$ states and hence we have at most $L=2$. Furthermore, the expectation value of $\hat{L}^{2}$ in diatomic molecules is generally only weakly $r$-dependent for a given electronic state. ${ }^{21}$ Hence the $\hat{L}^{2}$ term only induces a shift in the electronic energy in the order of $L(L$ $+1) \hbar^{2} / 2 \mu r^{2}$ and we neglect this contribution. The $\hat{L}^{ \pm} \hat{S}^{\mp} / 2 \mu r^{2}$ term couples states which are also coupled by 
SO coupling. However, because of the $1 / 2 \mu r^{2}$ factor it is much smaller than the SO coupling (see Paper I) and we neglect it. We also neglect the $\hat{L}^{ \pm} \hat{J}^{+}$term. This term couples states with different $\Lambda$ values, so its main effect would be to give (small) perturbations for nearly degenerate states of different electronic character, e.g., near crossings. We do keep the $\hat{S}^{ \pm} \hat{J}^{\mp}$ term, however, since it gives rise to intra state coupling. In particular, it couples the $\Omega=0^{-}, \pm 1$ components of the $A^{3} \Sigma_{u}^{+}$state. We will come back to this point in the discussion (Sec. III F).

To summarize we use a rotational Hamiltonian,

$$
\hat{H}_{\text {rot }}=\hat{H}_{\text {rot }}^{\text {(diag) }}+\hat{H}_{\text {rot }}^{\text {(JS) }}
$$

with

$$
\hat{H}_{\text {rot }}^{\text {(diag) }}=\frac{1}{2 \mu r^{2}}\left(\hat{J}^{2}+\hat{S}^{2}-\hat{J}_{z}^{2}-\hat{L}_{z}^{2}-\hat{S}_{z}^{2}\right)
$$

and

$$
\hat{H}_{\mathrm{rot}}^{(\mathrm{JS})}=\frac{-1}{2 \mu r^{2}}\left(\hat{S}^{+} \hat{J}^{-}+\hat{S}^{-} \hat{J}^{+}\right) .
$$

In the Appendix we define electronic-rotation Hund's case (a) basis functions $|(L) \Lambda S \Sigma J M \Omega ; r\rangle$, which are eigenfunctions of $\hat{H}_{\text {Coul }}$ [see Eq. (1)] as well as $\hat{H}_{\text {rot }}^{\text {(diag) : }}$

$$
\begin{gathered}
\left\{\hat{H}_{\mathrm{rot}}^{(\mathrm{diag})}-\left[J(J+1)+S(S+1)-\Omega^{2}-\Lambda^{2}-\Sigma^{2}\right]\right\} \\
\times|(L) \Lambda S \Sigma J M \Omega ; r\rangle=0 .
\end{gathered}
$$

The matrix elements of $\hat{H}_{\text {rot }}^{(\mathrm{JS})}$ follow directly from

$$
\begin{aligned}
& \hat{S}^{ \pm} \hat{J}^{\mp}|(L) \Lambda S \Sigma J M \Omega ; r\rangle \\
& \quad=c_{ \pm}(J, \Omega) c_{ \pm}(S, \Sigma)|(L) \Lambda S \Sigma \pm 1 J M \Omega \pm 1 ; r\rangle,
\end{aligned}
$$

where $c_{ \pm}(l, m)=[l(l+1)-m(m \pm 1)]^{1 / 2}$. In the Appendix we show that states of parity $p= \pm 1$, containing an ungerade electronic part, can be constructed as

$$
\begin{aligned}
|(L) \Lambda S \Sigma J M \Omega p ; r\rangle= & \frac{1}{\sqrt{2\left(1+\delta_{\Lambda, 0} \delta_{\Sigma, 0}\right)}}[|(L) \Lambda S \Sigma J M \Omega ; r\rangle \\
& -p(-1)^{J}|(L)-\Lambda S-\Sigma J M-\Omega ; r\rangle .
\end{aligned}
$$

\section{B. Vibrational motion}

The vibrational motion is treated by a sinc-function discrete variable representation $^{22}$ (sinc-DVR). The localized radial basis functions $\phi_{n}(r)=\langle r \mid n\rangle$ are associated with the grid points $r_{n}=r_{0}+n \Delta$, where $\Delta$ is the grid spacing, via

$$
\phi_{n}(r)=\frac{1}{\sqrt{\Delta}} \operatorname{sinc}\left(\pi \frac{r-r_{n}}{\Delta}\right),
$$

where $\operatorname{sinc}(x)=\sin (x) / x$. These functions are orthonormal. The matrix elements of $\hat{H}_{\text {vib }}$ are given by

$$
\left\langle n\left|\hat{H}_{\mathrm{vib}}\right| n^{\prime}\right\rangle=\left\{\begin{array}{l}
\frac{\hbar^{2}}{2 \mu} \frac{\pi^{2}}{3 \Delta^{2}}, \quad n=n^{\prime}, \\
\frac{\hbar^{2}}{2 \mu} \frac{2}{\Delta^{2}} \frac{(-1)^{n-n^{\prime}}}{\left(n-n^{\prime}\right)^{2}}, \quad n \neq n^{\prime} .
\end{array}\right.
$$

In a DVR all multiplicative operators are represented by diagonal matrices, so for the potential matrix elements we have

$$
\left\langle n\left|V_{L|\Lambda| S}(r)\right| n^{\prime}\right\rangle=\delta_{n, n^{\prime}} V_{L|\Lambda| S}\left(r_{n}\right) .
$$

When evaluating rotational Hamiltonian matrix elements we may use

$$
\left\langle n\left|\frac{1}{2 \mu r^{2}}\right| n^{\prime}\right\rangle=\delta_{n, n^{\prime}} \frac{1}{2 \mu r_{n}^{2}} .
$$

Our RVE basis functions are products:

$$
|(L) \Lambda S \Sigma J M \Omega n\rangle \equiv|(L) \Lambda S \Sigma J M \Omega ; r\rangle \phi_{n}(r) .
$$

Since we neglect the electronic radial derivative coupling for Hund's case (a) basis functions, the vibrational Hamiltonian matrix is diagonal in all angular quantum numbers.

The total dimension of the basis that is required to converge all the states up to the dissociation limit is quite large (order $10^{4}$ ). Therefore we follow a two-step procedure in which we exploit the fact that $\hat{H}_{\text {rot }}^{(\mathrm{JS})}$ is the only term in the Hamiltonian that couples different $\Omega$ values. Thus for each value of $\Omega$ we compute and diagonalize the Hamiltonian matrix of

$$
\hat{H}_{0}=\hat{H}_{\mathrm{Coul}}+\hat{H}_{\mathrm{SO}}+\hat{H}_{\mathrm{vib}}+\hat{H}_{\mathrm{rot}}^{\text {(diag) }} .
$$

Since $\hat{H}_{0}$ does not lift the degeneracy of odd and even parity states, we solve this problem in a parity unadapted basis with $\Omega \geqslant 0$ and we obtain the eigenfunctions as

$$
|J \Omega v\rangle=\sum_{\substack{L \Lambda S \Sigma n \\(\Lambda+\Sigma=\Omega)}}|(L) \Lambda S \Sigma J M \Omega n\rangle c_{L \Lambda S \Sigma n}^{(J \Omega v)} .
$$

Note that for $\Omega=0$ these eigenfunctions have an intrinsic parity. These functions are labeled $\Omega=0^{+}$for $p=+1$ or $\Omega$ $=0^{-}$for $p=-1$. For $\Omega \neq 0$ we obtain parity adapted functions as

$$
|J \Omega v p\rangle=\sum_{L \Lambda S \Sigma n}|(L) \Lambda S \Sigma J M \Omega p n\rangle c_{L \Lambda S \Sigma n}^{(J \Omega v)} .
$$

We will use the conventional $e / f$ parity label which corresponds to $p(-1)^{J}$ being even $(e)$ and odd $(f)$, respectively [see Eq. (10)]. For the ${ }^{16} \mathrm{O}^{16} \mathrm{O}$ molecule one can show that the ungerade states must have odd parity $(p=-1)$ (see the Appendix) and hence for even $J$ only $f$ states exist and for odd $J$ only $e$ states. Also note that $0_{u}^{+}$states must have $e$ parity (and hence only occur for odd $J$ ) and $0_{u}^{-}$states must have $f$ parity (and hence occur only for even $J$ ).

In the final step of the calculation we select all (odd parity) eigenfunctions of $\hat{H}_{0}$ which have an energy $E$ that is less than a certain threshold ( $\left.E_{\text {thresh }}\right)$ and we use these functions as a basis to diagonalize the total Hamiltonian $\hat{H}=\hat{H}_{0}$ 


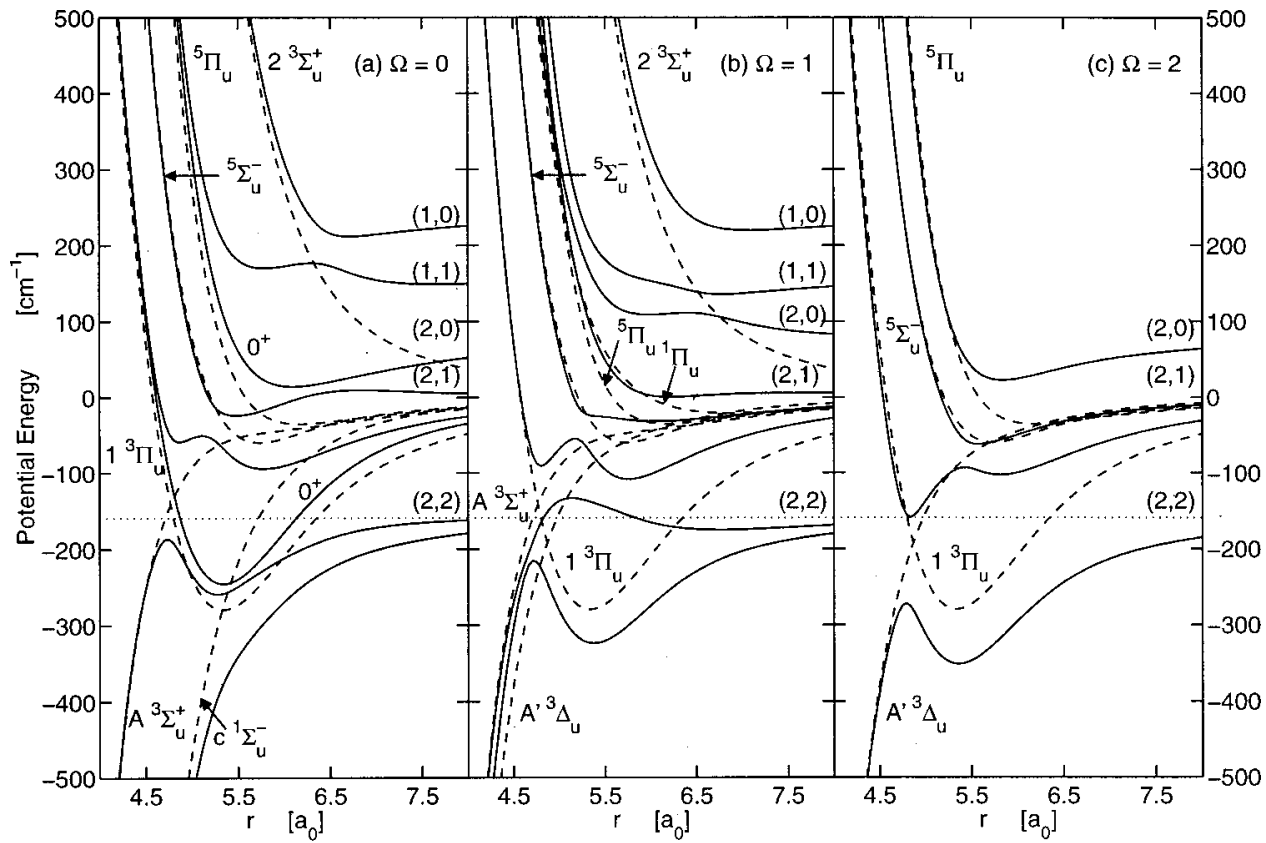

FIG. 1. Hund's case (a) (dashed lines) and (c) (solid lines) potential energy curves for $\Omega=0,1$, and 2 in part (a), (b), and (c), respectively. In part (a), case (c) $\Omega=0^{+}$curves have been marked $0^{+}$, other (not marked) curves are $0^{-}$. Hund's case (a) labels are formally only applicable to the dashed lines. The dotted line represents the $\mathrm{O}\left({ }^{3} P_{2}\right)+\mathrm{O}\left({ }^{3} P_{2}\right)$ dissociation limit, the different case $(\mathrm{c})$ dissociation limits $\mathrm{O}\left({ }^{3} P_{j_{a}}\right)+\mathrm{O}\left({ }^{3} P_{j_{b}}\right)$ are marked $\left(j_{a}, j_{b}\right)$.
$+\hat{H}_{\mathrm{rot}}^{(\mathrm{JS})}$. Convergence of the calculations is checked by comparing the eigenvalues of $\hat{H}$ for several values of $E_{\text {thresh }}$.

For a given set of potential energy curves and spin-orbit couplings this procedure gives essentially exact results. However, since we cannot expect our $a b$ initio calculations to be accurate to spectroscopic resolution, we need a thorough understanding of the spectrum in order to convincingly argue that a new assignment is called for. For this purpose we also report the results of approximate calculations in which we ignore the rotational part of the Hamiltonian $\left(\hat{H}_{\text {rot }}\right)$ and treat the molecule as either a pure Hund's case (a) or (c). In the Hund's case (a) calculations we use a Hamiltonian that includes $\hat{H}_{\text {Coul }}, \hat{H}_{\text {vib }}$, and the part of $\hat{H}_{\text {SO }}$ that is diagonal in $(L), \Lambda$ (and $\Sigma$ ) [see Eq. (3)]. For the Hund's case (c) calculation we first diagonalize $\hat{H}_{\mathrm{Coul}}+\hat{H}_{\mathrm{SO}}$ in the electronic basis for each point of the radial grid to obtain Hund's case (c) potentials. Subsequently, we take into account the vibrational Hamiltonian in a Born-Oppenheimer type approximation, i.e., treating the system as a set of independent onedimensional vibrational problems. To compare with these approximate calculations we also report a full calculation, with all of the electronic couplings included, but with neglect of the rotational Hamiltonian.

\section{Convergence of the sinc-DVR}

In the DVR calculation we employed a grid ranging from $r=1.6 a_{0}$ to $r=27 a_{0}$ with a grid spacing of $\Delta r$ $=0.045 a_{0}$. For the most strongly bound state in our study, the $c^{1} \Sigma_{u}^{-}$state, which has a $D_{e}$ of $8999 \mathrm{~cm}^{-1}$, this $\Delta r$ corresponds to 4 points per de Broglie wavelength, which we computed as $2 \pi\left(2 \mu D_{e}\right)^{-1 / 2}$. We checked that the convergence with respect to $\Delta r$ of even the highest vibrational level $(v=19)$ of the $c$ state is better than $4 \times 10^{-7} \mathrm{~cm}^{-1}$. The innermost point of the grid at $1.6 a_{0}$ is chosen well into the repulsive region of all potentials involved and the results are fully converged with respect to this parameter. The very large grid size guarantees that even states located at only about $1 \mathrm{~cm}^{-1}$ below the dissociation limit are converged to better than $10^{-3} \mathrm{~cm}^{-1}$.

\section{DISCUSSION}

\section{A. Potentials}

In Fig. 1 we show the ABO potentials (the dashed lines) and the Hund's case (c) potentials (the solid lines) for $\Omega=0,1$, and 2. Details of the $a b$ initio calculations of the potentials and the spin-orbit couplings used to construct the Hund's case (c) potentials, as well as the fits can be found in Paper I. In Table I we report the spectroscopic parameters $r_{e}, D_{e}$, and $\omega_{e}$ for these potentials. The $D_{e}$ is computed with respect to the $\mathrm{O}\left({ }^{3} P_{2}\right)+\mathrm{O}\left({ }^{3} P_{2}\right)$ dissociation limit (dotted line in Fig. 1) which lies $159 \mathrm{~cm}^{-1}$ below the asymptotic value of the ABO potentials. Note that in the Hund's case (c) description local minima of the $\Omega=0^{-}, 1$, and 2 potentials correspond to the $1^{3} \Pi_{u, \Omega}$ states. In Table I we also give the barriers to the inner well which supports the Herzberg states.

In Fig. 2 we show the $r$-dependent diagonal spin-orbit matrix element $A_{\Omega}(r)=\left\langle 1^{3} \Pi_{u, \Omega}\left|\hat{H}_{\mathrm{SO}}\right| 1^{3} \Pi_{u, \Omega}\right\rangle$. In a Hund's

TABLE I. Spectroscopic parameters $D_{e}$ and $\omega_{e}\left(\right.$ in $\mathrm{cm}^{-1}$ ) and $r_{e}$ (in $a_{0}$ ) for Hund's case (a) and (c) $1{ }^{3} \Pi_{u, \Omega}$ potentials. For the case (c) potentials we also report the position $r$ (in $a_{0}$ ) of the barrier between the inner and outer well and its height $\left(\right.$ in $\mathrm{cm}^{-1}$ ) relative to the minimum of the outer well at $r=r_{e}$.

\begin{tabular}{cccccccc}
\hline \hline & & & & & \multicolumn{2}{c}{ Barrier } \\
\cline { 6 - 8 } Case & $\Omega$ & $r_{e}$ & $D_{e}$ & $\omega_{e}$ & $r$ & Height \\
\hline (a) & $0,1,2$ & 5.33 & 120.4 & 92.5 & - & - \\
(c) & $0^{-}$ & 5.27 & 100.0 & 91.7 & 4.73 & 73.0 \\
(c) & 1 & 5.37 & 164.6 & 83.0 & 4.72 & 109.0 \\
(c) & 2 & 5.36 & 192.0 & 85.4 & 4.79 & 79.4 \\
\hline \hline
\end{tabular}




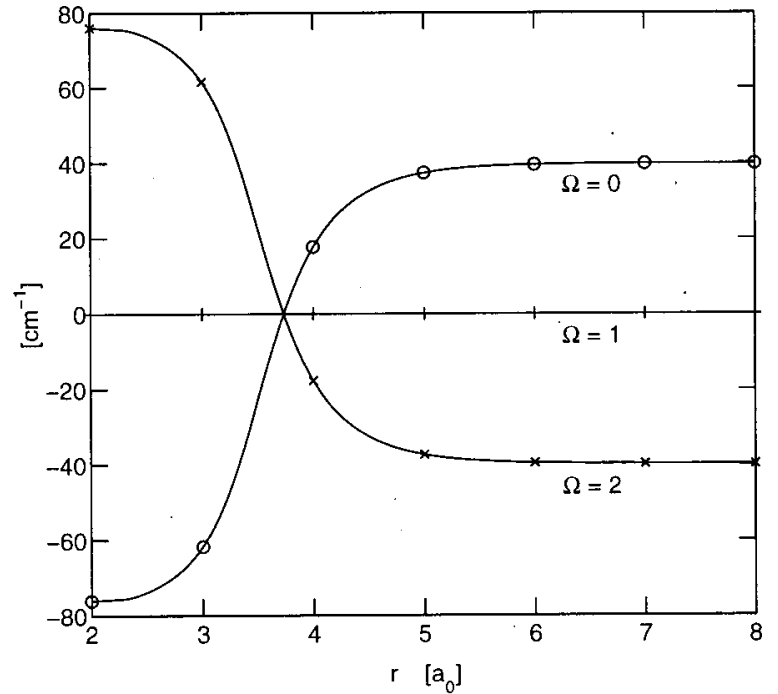

FIG. 2. The diagonal spin-orbit matrix elements $\left\langle 1{ }^{3} \Pi_{u, \Omega}\right| \hat{H}_{\mathrm{SO}}(r)$ $\left|1^{3} \Pi_{u, \Omega}\right\rangle$, for $\Omega=0,1$, and 2 .

case (a) description the multiplet splittings of the $1^{3} \Pi_{u, \Omega}$ components is determined by $A_{\Omega}(r)=A(r) \Lambda \Sigma=A(r)(\Omega$ $-1)$. If the dominant configuration of the $1^{3} \Pi_{u}$ state is $1 s^{4} 2 s^{4} 2 p \sigma_{g}^{2} 2 p \pi_{u}^{4} 2 p \pi_{g} 2 p \sigma_{u}$ one expects a regular multiplet, i.e., $A(r)>0$ (see Table 30 in Ref. 2). Figure 2 shows that for $r<3.8 a_{0}$ this is the case. However, for larger internuclear separations, which are relevant for the $1^{3} \Pi_{u}$ state $\left(r \approx 5.5 a_{0}\right)$, the $a b$ initio calculation shows that such a simple description no longer applies.

\section{B. Rotationless levels}

In Fig. 3 we show the results of all our bound state calculations in which the rotational part of the Hamiltonian was ignored. At the right hand side of the figure we show the levels observed by Jenouvrier et al. and the original assignment.

(a) Case (a). When we treat the $1^{3} \Pi_{u}$ state in the Hund's case (a) approximation described in the theory section we find the levels shown in the first column of Fig. 3. Note that the zero of energy in this plot corresponds to the
$\mathrm{O}\left({ }^{3} P_{2}\right)+\mathrm{O}\left({ }^{3} P_{2}\right)$ dissociation limit and hence these approximate Hund's case (a) "bound" levels may have a positive energy up to $159 \mathrm{~cm}^{-1}$. As expected, we find an inverted multiplet in the Hund's case (a) approximation.

(b) Case (c). We also approximated the bound levels by solving one-dimensional vibrational problems employing the Hund's case (c) potentials. The results are shown in the second column in Fig. 3. Note that the $\Omega$ components of the $1^{3} \Pi_{u, \Omega}$ are not equally spaced, in contrast with the Hund's case (a) description. In the Hund's case (c) description the $1^{3} \Pi_{u}(v)$ states can mix with the Herzberg states by tunneling through the barrier between the inner and outer wells in the potentials. In Fig. 4 we plot the vibrational wave functions corresponding to the five case (c) energy levels shown in Fig. 3. Clearly the $v=0$ states are sufficiently well localized in the outer well to allow an unambiguous assignment. The $1^{3} \Pi_{u, 1}(1)$ level lies also below the $(\Omega=1)$ barrier. The $\Omega=2$ barrier is lower and the $1^{3} \Pi_{u, 2}(1)$ level lies above this barrier, and is rather strongly mixed with the $A^{\prime}{ }^{3} \Delta_{u, 2}(12)$ level.

(c) Coupled calculation. In the third column of Fig. 3 we show the results of a fully coupled calculation. Since we still left out the rotational part of the Hamiltonian, $\Omega$ is a good quantum number. These levels are computed by taking all electronic Hund's case (a) basis functions for a specific value of $\Omega$ [see Eqs. (2) and (3)], combining them with the sincDVR basis functions [Eq. (11)] to describe the vibrational motion [Eq. (12)] and diagonalizing $\hat{H}_{\mathrm{Coul}}+\hat{H}_{\mathrm{SO}}+\hat{H}_{\mathrm{vib}}$ in this basis. Again, mixing with the Herzberg states occurs, so we had to inspect the wave functions to assign the levels. Compared to the case (c) approximation only small shifts occur. In particular, the $v=0, \Omega=0$ level shifts from 0.8 $\mathrm{cm}^{-1}$ below the $v=1, \Omega=1$ level to $7 \mathrm{~cm}^{-1}$ above it.

\section{New assignment}

We are now in a position to make a first comparison between our computed levels and the experimentally observed levels. Although Jenouvrier et al. assumed a regular Hund's case (a) they had to assign the lowest observed perturbations to a $\Omega \neq 01^{3} \Pi_{u}(0)$ level because the $A^{\prime}{ }^{3} \Delta_{u, 2}(12)$ state was involved (and ${ }^{3} \Delta_{2}$ does not couple with ${ }^{3} \Pi_{0}$ ).

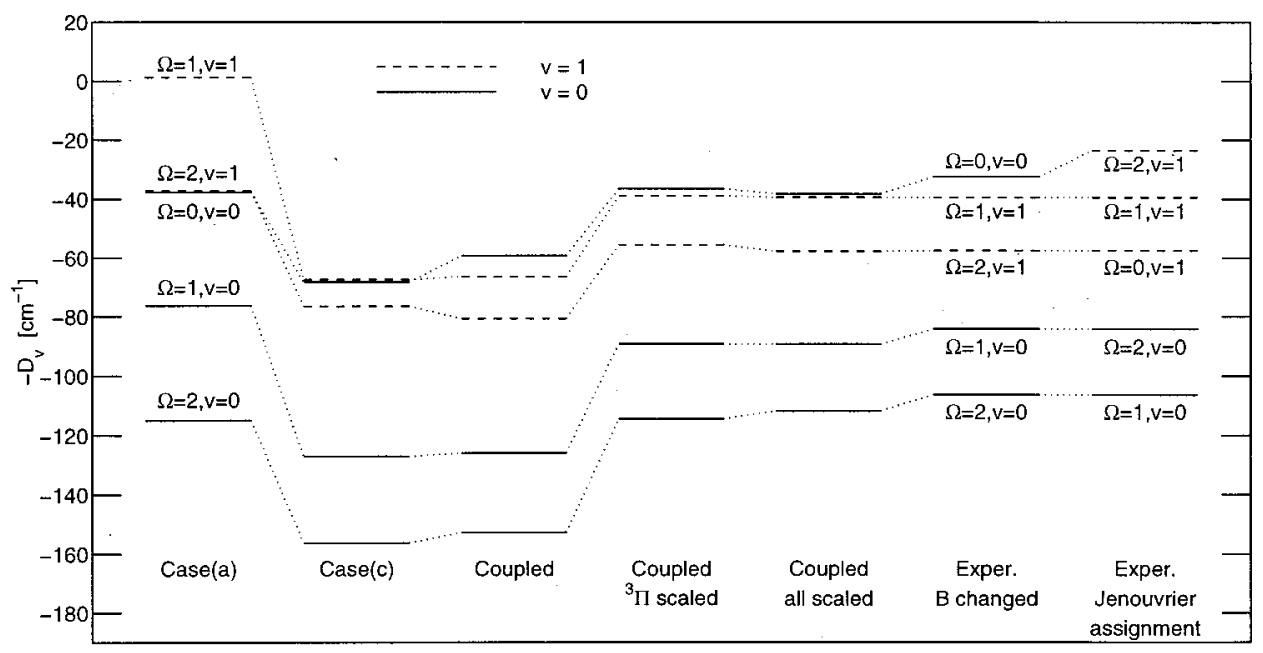

FIG. 3. Bound state calculations for the $1{ }^{3} \Pi_{u}$ vibrational levels where the rotational part of the Hamiltonian was ignored. From left to right approximate Hund's case (a), approximate Hund's case (c), full coupled calculation, coupled calculation with scaled ${ }^{3} \Pi_{u}$ potential, coupled calculation where both the $1{ }^{3} \Pi_{u}$ and Herzberg potentials are scaled, experimental results, with our model rotational constant for the highest level (see the text) and our new assignment, and original experimental results with original assignment. 


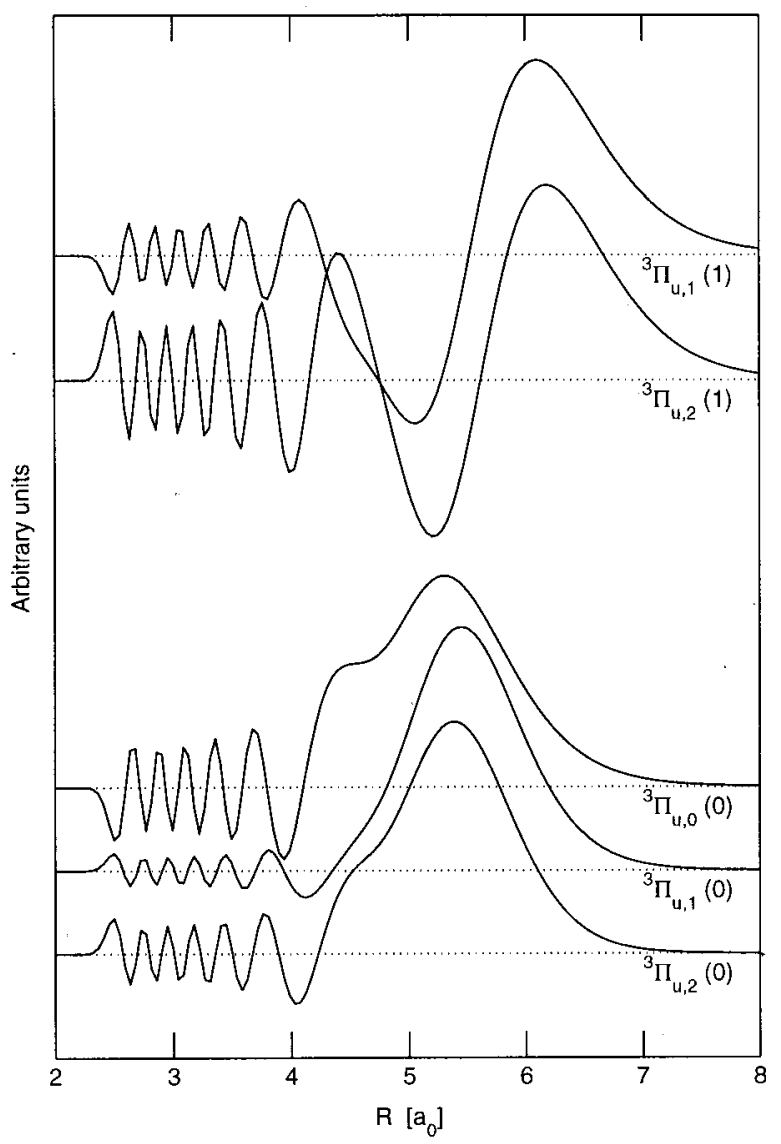

FIG. 4. Vibrational wave functions for the $1{ }^{3} \Pi_{u, \Omega}(v)$ levels calculated in the Hund's case (c) approximation.

Hence we assume that our lowest two levels, with $v=0$ and $\Omega=2,1$ correspond to the lowest two observed states. In Sec. III F we will show that reversing the $(v=0) \Omega=1$ and $\Omega=2$ assignment is not at all inconsistent with the observations.
The next two observed $1{ }^{3} \Pi_{u}$ levels had a distinctly smaller rotational constant and were assigned $v=1$, which is consistent with the ordering of the computed levels. However, we find that the lowest $v=11^{3} \Pi_{u}$ level has $\Omega=2$ instead of $\Omega=0$. In the experiment, the lowest $v=1$ level causes perturbations in both the $F_{1 f}$ and $F_{2 e}$ components of the $A^{3} \Sigma_{u, 1}^{+}(11)$ states, which is consistent with our $\Omega=2$ assignment.

For the highest observed $1{ }^{3} \Pi_{u}$ level, which was assigned $v=1, \Omega=2$ by Jenouvrier et al., only one perturbation [of the $J=14 F_{3 f} A^{3} \Sigma_{u}^{+}(11)$ level] was observed, and hence no direct determination of its rotational constant was possible. The assignment is only logical if one assumes a regular Hund's case (a) for the $v=1$ state. As we will see below, this observed $(J=14)$ perturbation can be very well explained by our $v=0, \Omega=0$ level which lies just above the $v=1, \Omega=1$ level. Assigning this $J=14$ perturbation to a $v$ $=0$ level instead of a $v=1$ level results in a different $(J$ $=0$ ) term value because the rotational constants of $v=0$ and $v=1$ states are different. In Sec. III F we will show how this new assignment leads to a new "observed" term value for the $1^{3} \Pi_{u, 0^{-}}(0)$ level. In column 6 of Fig. 3 we show the experimental data with the adapted $1{ }^{3} \Pi_{u, 0^{-}}$level.

\section{Adjusting the $1^{3} \Pi_{u}$ curve}

In the experiment information about the $1{ }^{3} \Pi_{u}$ levels is obtained from a characteristic pattern of perturbations in a plot of the term values of the observed (Herzberg) levels as a function of $J(J+1)$ (see Fig. 4 in Ref. 19). In order to validate our assignment we construct in Sec. III F a similar plot using computed RVE levels (Fig. 5). In order to allow a meaningful comparison between experiment and theory it is important that the perturbations between the highly vibrationally excited Herzberg states and the $1{ }^{3} \Pi_{u}$ states occur at (approximately) the same value of $J$. Clearly, such a near

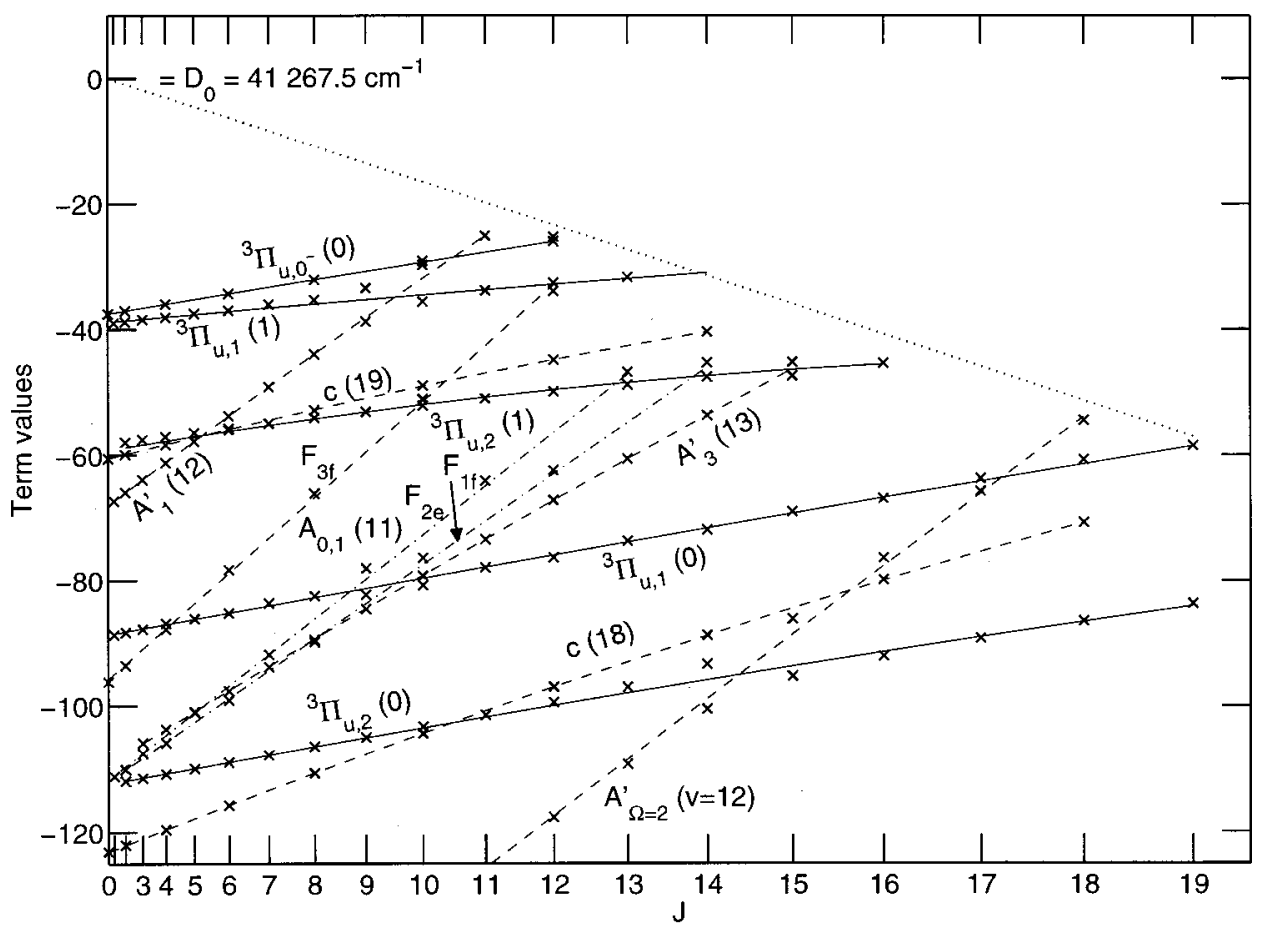

FIG. 5. Calculated RVE energy levels (x marks) lying less than $120 \mathrm{~cm}^{-1}$ below the dissociation limit (dotted line). For clarity, $0.15 J(J+1) \mathrm{cm}^{-1}$ was subtracted from the term values. Solid lines are fitted values $T_{v, \Omega}(J)$ $=-D_{v, \Omega}+B_{v, \Omega} J(J+1)-\widetilde{D}_{v, \Omega}[J(J$ $+1)]^{2}$ for the $1{ }^{3} \Pi_{u}$ levels, and dashed lines are the same fits for the Herzberg levels. 
TABLE II. Position of the $1{ }^{3} \Pi_{u, \Omega}(v)$ levels with different combinations of $\alpha$ and $r_{0}$ in the adapted $1{ }^{3} \Pi_{u}$ potential energy curve. Experimental data for $1{ }^{3} \Pi_{u, 0}(0)$ is given between parentheses because it depends on the erroneous experimental assignment as $v=1$.

\begin{tabular}{ccccccc}
\hline \hline & & \multicolumn{5}{c}{$D_{v}$} \\
\cline { 3 - 7 }$\alpha$ & $r_{0}$ & $1{ }^{3} \Pi_{u, 2}(0)$ & $1{ }^{3} \Pi_{u, 1}(0)$ & $1{ }^{3} \Pi_{u, 2}(1)$ & $1{ }^{3} \Pi_{u, 1}(1)$ & $1{ }^{3} \Pi_{u, 0^{-}}(0)$ \\
\hline \multicolumn{2}{c}{ Experiment } & 106.3 & 84.1 & 57.5 & 39.5 & $(23.5)$ \\
1.5655 & 0.00 & 119.7 & 94.7 & 59.5 & 42.1 & 37.1 \\
1.5655 & 0.13 & 114.4 & 89.2 & 55.6 & 39.0 & 36.5 \\
1.5655 & 0.26 & 101.5 & 83.0 & 51.3 & 35.6 & 35.8 \\
1.3246 & -0.693 & 101.0 & 82.5 & 51.0 & 35.4 & 35.1 \\
\multicolumn{2}{l}{ Original curve } & 152.8 & 125.9 & 80.6 & 66.4 & 59.3 \\
\hline \hline
\end{tabular}

perfect agreement between ab initio results and experiment is very difficult to achieve. Therefore, before we include the rotational part of the problem, we adjust the potentials slightly in order to shift the vibrational levels closer to the observed positions.

First, since our calculated $1{ }^{3} \Pi_{u}$ levels lie somewhat too deep and the $v=0 / v=1$ separation is somewhat too large, we add a simple two-parameter repulsive term $\exp [-\alpha(r$ $\left.\left.-r_{0}\right)\right]$ to the $1^{3} \Pi_{u} \mathrm{ABO}$ potential. In Table II we show the energies of the $1^{3} \Pi_{u, \Omega}(v)$ levels for four combinations of $\alpha$ and $r_{0}$, together with the experimental results and the results for the unadjusted potential (which were already shown in Fig. 3). Since the result with $\alpha=1.5655$ and $r_{0}=0.13$ gives agreement with all experimental data to within a few $\mathrm{cm}^{-1}$ we did not attempt further optimization of the parameters. The results with $\left(\alpha, r_{0}\right)=(1.5655,0.26)$ and $(1.3246$, -0.693 ) show that similar results can be obtained with different combinations of $\alpha$ and $r_{0}$. We chose to use $\alpha$ $=1.5655$ above $\alpha=1.3246$ because a larger $\alpha$ yields a smaller relative change in the short-range part of the potential when inducing an equal change in position of the bound levels. Note furthermore that for the $1{ }^{3} \Pi_{u, 0}(0)$ level the difference between calculated and experimental level position is $36.5-23.5=13 \mathrm{~cm}^{-1}$ if we take the original data from the Jenouvrier paper. However, using our model to derive the $J=0$ level from the observed $J=14$ level the agreement becomes much better: $36.5-32.4=4.1 \mathrm{~cm}^{-1}$.

TABLE III. Term values with respect to the dissociation limit, $D_{v}$, for the high-lying Herzberg vibrational levels. Calculated levels are coupled state calculation, where rotation has been neglected.

\begin{tabular}{lcccc}
\hline \hline & \multicolumn{3}{c}{ Calculated } & \\
\cline { 2 - 4 } Level & Orig. curve & $1^{3} \Pi_{u}$ scaled & All scaled & Exper. (Ref. 19) \\
\hline$A^{\prime}{ }^{3} \Delta_{u, 2}(12)$ & 111.3 & 104.9 & 168.7 & 175.3 \\
$A^{\prime}{ }^{3} \Delta_{u, 2}(11)$ & 321.8 & 321.3 & 404.8 & 397.9 \\
$A^{\prime}{ }^{3} \Delta_{u, 1}(11)$ & 211.3 & 210.8 & 292.1 & 279.8 \\
$A^{3} \Sigma_{u, 1}^{+}(11)$ & 54.0 & 56.4 & 112.2 & 108.5 \\
$A^{3} \Sigma_{u, 0^{-}}^{+}(11)$ & 39.8 & 42.2 & 97.5 & 95.6 \\
$c^{1} \Sigma_{u, 0^{-}}^{+}(19)$ & 40.4 & 36.5 & 61.0 & 67.0 \\
$c^{1} \Sigma_{u, 0^{-}}^{-}(18)$ & 95.4 & 82.9 & 123.5 & 130.2 \\
$c^{1} \Sigma_{u, 0^{-}}^{-}(17)$ & 170.8 & 163.9 & 240.3 & 242.6 \\
\hline \hline
\end{tabular}

TABLE IV. Parameter values used in the adaptation of the Herzberg Coulombic potential energy curves to experiment, according to Eq. (19).

\begin{tabular}{cccc}
\hline \hline State & $f_{\text {vert }}$ & \multicolumn{1}{c}{$r_{0}$} & $f_{\text {hor }}$ \\
\hline$A^{\prime}{ }^{3} \Delta_{u}$ & 1.01906 & 2.878636 & 0.9855 \\
$A^{3} \Sigma_{u}^{+}$ & 1.01751 & 2.892466 & 0.9845 \\
$c^{1} \Sigma_{u}^{u}$ & 1.02091 & 2.8880295 & 0.9800 \\
\hline \hline
\end{tabular}

\section{E. Scaling the Herzberg curves}

In the next step we adjusted the three Herzberg $\mathrm{ABO}$ potentials $\left(A^{\prime 3} \Delta_{u}, A^{3} \Sigma_{u}^{+}, c^{1} \Sigma_{u}^{-}\right)$according to

$$
V^{\text {new }}(r)=f_{\text {vert }} V\left[r_{0}+\left(r-r_{0}\right) f_{\text {hor }}\right] \text {. }
$$

Since the computed rotational constants of the lowest Herzberg vibrational levels were already in very good agreement with experiment (better than $0.7 \%$, see Paper I) we took, for each curve, $r_{0}$ equal to the expectation value of $r$ for the lowest vibrational level. This ensures that the scaling has a negligible effect on these rotational constants. The vertical scaling $f_{\text {vert }}$ was chosen to get exact agreement with experiment for $D_{e}$ in the Hund's case (c) approximation. Finally, $f_{\text {hor }}$ was varied until the highest vibrational levels were in agreement with experiment to within $6 \mathrm{~cm}^{-1}$. In Table III we report the $D_{v}$ for several high lying Herzberg vibrational levels for the original curves as well as the adjusted curves, together with the experimental data (taken from Ref. 19). Note the influence of the scaling of the $1^{3} \Pi_{u}$ ABO curve on the Herzberg levels: the $A^{\prime}{ }^{3} \Delta_{u}$ and $c{ }^{1} \Sigma_{u}^{-}$ levels go up and the $A^{3} \Sigma_{u}^{+}$levels go down, resulting in a reversal of $A^{3} \Sigma_{u, 0^{-}}^{+}(11)$ and $c^{1} \Sigma_{u, 0^{-}}^{-}(19)$. The parameters are given in Table IV. Because of the mixing of the Herzberg states with the $1{ }^{3} \Pi_{u}$ states the latter are slightly altered by the change in the Herzberg potentials, as shown in the fifth column of Fig. 3.

\section{F. Rotational energy levels}

In Fig. 5 we plot the RVE energy levels as a function of $J(J+1)$. The levels were calculated with the two-step procedure described in Sec. II B, employing the scaled $1{ }^{3} \Pi_{u}$ and Herzberg $\mathrm{ABO}$ potentials and taking into account the rotational Hamiltonian of Eq. (5). The threshold for selecting basis functions in the second step of the calculation was set to $E_{\text {thresh }}=24.2 \mathrm{~cm}^{-1}$ above the $\mathrm{O}\left({ }^{3} P_{2}\right)+\mathrm{O}\left({ }^{3} P_{2}\right)$ dissociation limit. The dimension of the resulting basis ranges from 59 for $J=0$ (only $\Omega=0$ states) via 275 for $J=3$ (all $\Omega$ components) to 205 for $J=19$. In changing $E_{\text {thresh }}$ from 19.2 $\mathrm{cm}^{-1}$ to $24.2 \mathrm{~cm}^{-1}, 2,15$, and 19 extra basis functions were selected for $J=0,3$, and 19 , and all bound level positions changed less than $0.028 \mathrm{~cm}^{-1}$, so we expect to be at least converged up to $0.02 \mathrm{~cm}^{-1}$. To reduce the slopes of the lines $0.15 J(J+1)$ has been subtracted from all term values in Fig. 5 (exactly as in Fig. 4 of Ref. 19). The dissociation limit is represented by the dotted line.

There is good agreement between Fig. 5 and the experimental results (Fig. 4 in Ref. 19). Our calculated $c^{1} \Sigma_{u}^{-}(18,19)$ levels are about $7 \mathrm{~cm}^{-1}$ too high and the $1{ }^{3} \Pi_{u}(0)$ levels are about $6 \mathrm{~cm}^{-1}$ too deep. As a result the 
TABLE V. Spectroscopic parameters for $1{ }^{3} \Pi_{u}$ bound states. Experimental data for the $1^{3} \Pi_{u, 0^{-}}(0)$ level is given between parentheses because this data depends on the erroneous experimental assignment of this level as $v=1$

\begin{tabular}{|c|c|c|c|c|c|c|c|c|}
\hline \multirow[t]{2}{*}{ Level } & \multicolumn{3}{|c|}{$D_{v, \Omega}$} & \multicolumn{2}{|c|}{$B_{v, \Omega}$} & \multirow[t]{2}{*}{$B_{v, \Omega}^{\text {eff }}(J)$} & \multirow[t]{2}{*}{$J$} & \multirow{2}{*}{$\begin{array}{c}\widetilde{D}_{v, \Omega} \\
10^{-5} \mathrm{~cm}^{-1}\end{array}$} \\
\hline & Calc. & Exp. & Lit. $^{\mathrm{a}}$ & Calc. & Exp. & & & \\
\hline $1^{3} \Pi_{u, 2}(0)$ & 112.6 & 106.3 & 96.9 & 0.2329 & 0.221 & 0.2268 & 16 & 2.23 \\
\hline $1^{3} \Pi_{u, 1}(0)$ & 89.0 & 84.1 & 76.9 & 0.2333 & 0.226 & 0.2319 & 11 & 1.05 \\
\hline $1^{3} \Pi_{u, 2}(1)$ & 59.5 & 57.5 & 54.7 & 0.2267 & 0.165 & 0.2090 & 13 & 9.71 \\
\hline $1^{3} \Pi_{u, 1}(1)$ & 39.2 & 39.5 & 38.0 & 0.1911 & 0.170 & 0.1863 & 16 & 1.78 \\
\hline $1^{3} \Pi_{u, 0}(0)$ & 37.8 & $(23.5)$ & 30.2 & 0.2265 & $(0.175)$ & 0.2215 & 14 & 1.84 \\
\hline
\end{tabular}

${ }^{\mathrm{a}}$ For $1{ }^{3} \Pi_{u}$ potential from Ref. 18.

calculated perturbations of the Herzberg levels occur at values of the rotational quantum number $J$ that differ at most about 2 with experiment.

The selection rule for spin-orbit coupling is $\Delta \Omega=0$. We observe that perturbations between same- $\Omega$ states are larger than for states with different $\Omega$ quantum number. The latter are-in our Hamiltonian—only coupled via $\hat{H}_{\text {rot }}^{(\text {JS) }}$ [Eq. (7)]. Note that the most visible effect is to shift down the $F_{1 f}$ component of the $A^{3} \Sigma_{u, 1}^{+}$(11) level via first order coupling with the $A^{3} \Sigma_{u, 0^{-}}^{+}$(11) $F_{3 f}$ levels. A similar shift does not occur for the $A^{3} \Sigma_{u, 1}^{+} F_{2 e}$ level since there is no $A^{3} \Sigma_{u, 0}^{+}$state of $e$ parity (compare the two dash-dotted lines in Fig. 5).

A $\Delta \Omega=0$ perturbation occurs at $J=14,15(16,17$ in the experiment) between the $1^{3} \Pi_{u, 2}(0)$ and $A^{\prime}{ }^{3} \Delta_{u, 2}(12)$ states. In the original assignment of Jenouvrier et al. this was a $\Delta \Omega=1$ perturbation $\left[1^{3} \Pi_{u, 1}(0)-A^{\prime 3} \Delta_{u, 2}(12)\right]$. In Fig. 5, however, we find that the perturbation between $A^{\prime}{ }^{3} \Delta_{u, 2}(12)$ and $1^{3} \Pi_{u, 1}(0)$ (around $J=17$ ) is negligible. Similarly, the perturbation between $A^{3} \Sigma_{u, 1}^{+}(11)$ and $1{ }^{3} \Pi_{u, 1}(0)$ around $J$ $=9,10(11,12$ in the experiment) was a $\Delta \Omega=1$ perturbation in the original assignment.

The solid lines in Fig. 5 represent fits for the $J$-dependent term values of the 5 perturbing $1{ }^{3} \Pi_{u}$ levels:

$$
T_{v, \Omega}(J)=-D_{v, \Omega}+B_{v, \Omega} J(J+1)-\widetilde{D}_{v, \Omega}[J(J+1)]^{2} .
$$

The dashed lines represent similar fits for the Herzberg levels. The parameters are given in Table $\mathrm{V}$, together with the experimental results. Centrifugal distortion parameters $\widetilde{D}_{v, \Omega}$ were not reported in Ref. 19, probably since the observed range of $J$ values for each state was too limited to extract a reliable value. Note that the $D_{0, \Omega=1,2}$ dissociation energies agree to within $6 \mathrm{~cm}^{-1}$ with experiment and that for $v=1$ the agreement is even better. For the $1{ }^{3} \Pi_{u, 0^{-}}(0)$ level there seems to be a discrepancy of $14.3 \mathrm{~cm}^{-1}$. In our calculation the crossing of the $1^{3} \Pi_{u, 0^{-}}(0)$ with the $A^{3} \Sigma_{u, 0^{-}}^{+}(11) F_{3 f}$ level occurs at $J \approx 13$. Jenouvrier et al. observed a perturbation of the $J=14, F_{3 f}$ component of the $A^{3} \Sigma_{u, 0^{-}}^{+}(11)$ level. They assigned this perturbation to the $1^{3} \Pi_{u, 2}(1)$ level. However, no further perturbations arising from this " $1{ }^{3} \Pi_{u, 2}(1)$ " state were observed and hence the reported rotational constant of $0.175 \mathrm{~cm}^{-1}$ was derived from observations of other $v=11^{3} \Pi_{u}$ states via the Hund's case (a) model [Eq. (31) in Ref. 19]. Furthermore the reported dissociation energy of
$23.5 \mathrm{~cm}^{-1}$ was derived by extrapolating the observed $J$ $=14$ term value using the rotational constant of $0.175 \mathrm{~cm}^{-1}$. However, with our assignment of the perturbation to the $1^{3} \Pi_{u, 0^{-}}(0)$ level, extrapolating to $J=0$ using our rotational constant $B_{0,0}=0.2265 \mathrm{~cm}^{-1}$ and distortion constant $\widetilde{D}_{0,0}$ $=1.84 \times 10^{-5} \mathrm{~cm}^{-1}$ we arrive at an experimental $D_{0,0}$ of $32.4 \mathrm{~cm}^{-1}$, which is in good agreement with our calculated value of $37.8 \mathrm{~cm}^{-1}$.

Our calculated rotational constants are somewhat too large. However, in the experiment the centrifugal distortion constants were neglected. Therefore, we also computed effective rotational constants (Table V),

$$
B_{v, \Omega}^{\mathrm{eff}}(J)=B_{v, \Omega}-\widetilde{D}_{v, \Omega} J(J+1),
$$

for $J$ values in the region of the dominant perturbations. The agreement with these effective rotational constants is within about 2 to 3 standard deviations, again with the exception of the $1^{3} \Pi_{u, 0}(0)$ level.

In Table V we also report (in the column marked "Lit.") results of a calculation employing a literature ${ }^{18}$ potential energy curve for the $1^{3} \Pi_{u}$ state (and all other potentials and couplings from our own scaled results). Since this is a calculation where the rotational Hamiltonian was neglected, we have no rotational and distortion constants in this case. We see that the vibrational levels lie too high, from $9.6 \mathrm{~cm}^{-1}$ for $1{ }^{3} \Pi_{u, 2}(0)$ to $1.5 \mathrm{~cm}^{-1}$ for $1^{3} \Pi_{u, 1}(1)$, an accuracy comparable with our adjusted $1^{3} \Pi_{u}$ potential energy curve. In Table VI we give spectroscopic parameters for the three $1{ }^{3} \Pi_{u} \mathrm{ABO}$ potential energy curves. We see that the parameters for the literature curve and the present adjusted curve differ by a fairly large amount, though the final level positions are of a reasonably good quality for both curves. This indicates the importance of the spin-orbit coupling in this recoupling region. In Paper I we showed that the original unscaled $\mathrm{ABO}$ states yield better spectroscopic results for the

TABLE VI. Spectroscopic parameters for the three $a b$ initio ABO potential energy curves under comparison.

\begin{tabular}{lccc}
\hline \hline \multicolumn{1}{c}{ Curve } & $R_{e}\left(\mathrm{a}_{0}\right)$ & $\omega_{e}\left(\mathrm{~cm}^{-1}\right)$ & $D_{e}\left(\mathrm{~cm}^{-1}\right)$ \\
\hline Literature (Ref. 18) & 5.6426 & 65.4072 & 191.9923 \\
From paper I & 5.3328 & 92.5180 & 279.5888 \\
Present, adjusted & 5.4991 & 85.2712 & 223.6329 \\
\hline \hline
\end{tabular}


Herzberg states than the literature curves from Ref. 18. We would therefore expect the original curve to be better for the $1{ }^{3} \Pi_{u}$ state also, but it is not. The original curve was calculated employing an equally good (in the long range, $r$ $\geqslant 7.5 a_{0}$ ) or better (in the short range, $r \leqslant 6.5 a_{0}$ ) one-electron basis than the literature curve, and both curves were calculated on the multireference configuration interaction (MRCI) plus size consistency correction level of theory. Therefore we tentatively suggest that the internal contraction scheme of the MOLPRO MRCI program, employed in the calculation in paper I, is not as good as uncontracted MRCI for this weakly bound van der Waals-like state, though in general the internal contraction scheme gives good results for chemically bound systems.

\section{SUMMARY AND CONCLUSIONS}

In addition to the three Herzberg states, there are five ungerade states in $\mathrm{O}_{2}$ that correlate with the $\mathrm{O}\left({ }^{3} P\right)+\mathrm{O}\left({ }^{3} P\right)$ dissociation limit. We calculated all rotational-vibrationalelectronic bound states up to $J=19$ supported by these potentials, taking into account spin-orbit and rotational couplings. We neglect the homogeneous spin-electronic $\left(\hat{L}^{ \pm} \hat{S}^{\mp} / 2 \mu r^{2}\right)$ and the $L$-uncoupling operator $\left(\hat{L}^{ \pm} \hat{J}^{\mp} / 2 \mu r^{2}\right)$, but we kept the $S$-uncoupling $\left(\hat{S}^{ \pm} \hat{J}^{\mp} / 2 \mu r^{2}\right)$. Ab initio potentials and SO couplings were available from our previous study.

In a recent spectroscopic study of the Herzberg bands perturbations were found in the $A^{3} \Sigma_{u}^{+}(v=11), c^{1} \Sigma_{u}^{-}(18$, 19), and $A^{\prime}{ }^{3} \Delta_{u}(12)$ levels. Assuming a regular Hund's case (a) multiplet for the $1^{3} \Pi_{u}$ state, these perturbations were assigned to the $1{ }^{3} \Pi_{u, 1}(0), 1^{3} \Pi_{u, 2}(0), 1{ }^{3} \Pi_{u, 0}(1), 1{ }^{3} \Pi_{u, 1}(1)$, and $1^{3} \Pi_{u, 2}(1)$ levels. Our calculations yield a new assignment of the perturbing levels, namely $1^{3} \Pi_{u, 2}(0), 1^{3} \Pi_{u, 1}(0)$, $1{ }^{3} \Pi_{u, 2}(1), 1{ }^{3} \Pi_{u, 1}(1)$, and $1{ }^{3} \Pi_{u, 0^{-}}(0)$. This new assignment is consistent with the experimental data, and better than the original assignment, because all the large perturbations are now explained by large $\Delta \Omega=0$ spin-orbit couplings, and the smaller perturbations by smaller $\Delta \Omega= \pm 1$ spin-rotation couplings. The calculated diagonal $1{ }^{3} \Pi_{u} \mathrm{SO}$ coupling is negative for $r \geqslant 4 a_{0}$ so one expects to find an inverted multiplet for the $1^{3} \Pi_{u}$ state, which has an $r_{e} \approx 5.3 a_{0}$. In this region the SO couplings are comparable in size to the Coulomb splittings between the eight ungerade states and a pure Hund's case description is not possible. An approximate Hund's case (c) description is in reasonable agreement with the more exact calculations.

We also slightly scaled the Herzberg potentials and adjusted the $1^{3} \Pi_{u}$ potential by adding a small repulsive term. In this way we achieved agreement with the experimentally observed perturbing levels to within $7 \mathrm{~cm}^{-1}$. Replacing the ab initio potential from Ref. 12 by the potential from Ref. 18 gave a similar good agreement (in combination with the scaled Herzberg potentials).

Our calculated rotational constants of the $1^{3} \Pi_{u}$ levels are slightly too large, but still agree with experiment within 2-3 standard deviations (see Table V), except for the level we assigned as $1^{3} \Pi_{u, 0^{-}}(0)$. This level was originally assigned as $1^{3} \Pi_{u, 2}(1)$ and we show that the reported rotational con- stant is an artifact of this incorrect assignment. We also compute rotational distortion constants and show that they are not negligible for the higher rotational levels that were observed experimentally.

\section{APPENDIX: BASIS FUNCTIONS AND ROTATIONAL HAMILTONIAN}

The recipes for computing rotational Hamiltonian matrix elements for Hund's case (a) and (c) basis functions can be found in several textbooks. ${ }^{21,23,24}$ The rules can be derived taking into account the normal and anomalous commutation relations of the appropriate rigid rotor angular momentum operators. This method was introduced by van Vleck ${ }^{25}$ for nonlinear molecules. For linear molecules the derivation is more difficult since two-angle embedded rotation operators have complicated commutation relations. ${ }^{20,26}$ The problem arises because a linear molecule only uniquely defines a BF $z$-axis. It was shown by Hougen ${ }^{20}$ that the familiar results can be obtained by the introduction of an extraneous rotation angle. This leads to an isomorphic Hamiltonian, for which only some of the eigenvalues and eigenfunctions correspond to the physical solutions. An analogous problem occurs in the study of van der Waals complexes when a two-angle embedded BF frame is chosen. In an effort to avoid the isomorphic Hamiltonian in that case alternative derivations were presented, one starting with Cartesian coordinates and applying the chain rule ${ }^{27}$ and one employing the Podolsky form of the Laplacian. ${ }^{28}$ Both derivations require a somewhat ad hoc rewriting of the Hamiltonian in terms of angular momentum operators to arrive at the familiar results and electron spin was not considered.

Here we present a new derivation which only requires elementary angular momentum theory and which does not involve the isomorphic Hamiltonian. Although we take the present $\mathrm{O}_{2}$ system as an illustration, our derivation is also completely rigorous for half-integer spin and the application to van der Waals complexes should be transparent. Furthermore we present a compact and rigorous derivation of the inversion symmetry behavior of the basis functions. Because several phase conventions have been used in the literature ${ }^{29}$ great care is required when applying textbook formulas in combination with $a b$ initio data.

The present approach was inspired by the discussion of angular momentum theory in Chap. 3 of the book by Biedenharn and Louck. ${ }^{30}$

\section{Basis functions}

The coordinates of the unit vectors that define the BF axes with respect to the space fixed (SF) frame are given by [Eq. (2.37) in Ref. 30]

$$
\begin{aligned}
{\left[\underline{e}_{x}^{\mathrm{BF}} \underline{e}_{y}^{\mathrm{BF}} \underline{e}_{z}^{\mathrm{BF}}\right] } & \equiv \mathbf{R}(\alpha, \beta, 0)=\mathbf{R}_{Z}(\alpha) \mathbf{R}_{Y}(\beta) \\
& =\left[\begin{array}{ccc}
\cos \alpha \cos \beta & -\sin \alpha & \cos \alpha \sin \beta \\
\sin \alpha \cos \beta & \cos \alpha & \sin \alpha \sin \beta \\
-\sin \beta & 0 & \cos \beta
\end{array}\right],
\end{aligned}
$$

where $\alpha$ and $\beta$ are the spherical polar coordinates of the 
diatomic internuclear axis with respect to the SF frame. We define two-angle embedded Hund's case (a) basis functions as

$$
\begin{aligned}
\mid(L) & \Lambda S \Sigma J M \Omega ; r\rangle \\
& \equiv \sqrt{\frac{2 J+1}{4 \pi}} D_{M \Omega}^{(J) *}(\alpha, \beta, 0) \hat{R}(\alpha, \beta, 0)|(L) \Lambda S \Sigma ; r\rangle_{\mathrm{SF}},
\end{aligned}
$$

where we introduced the rotation operator in the active convention

$$
\begin{aligned}
\hat{R}(\alpha, \beta, 0) & \equiv \hat{R}_{Z}(\alpha) \hat{R}_{Y}(\beta) \\
& =\exp \left(-i \alpha \hat{J}_{\text {elec }, Z}^{\mathrm{SF}}\right) \exp \left(-i \beta \hat{J}_{\text {elec }, Y}^{\mathrm{SF}}\right) .
\end{aligned}
$$

The total electronic angular momentum operator is defined as $\underline{\hat{J}}_{\text {elec }}^{\mathrm{SF}}=\hat{\hat{L}}^{\mathrm{SF}}+\underline{\hat{S}}^{\mathrm{SF}}$. The electronic wave functions calculated in paper I for the $\mathrm{O}$ atoms on the $\mathrm{SF} Z$-axis are denoted here as $|(L) \Lambda S \Sigma ; r\rangle_{\mathrm{SF}}$. Applying the rotation operator to these functions yields the $\mathrm{BF}$ electronic wave functions. The nuclear rotational part of the wave function is given by the Wigner D-matrix, which is also defined in the active convention. ${ }^{30,31}$ From here on we will write $D_{M \Omega}^{(J) * \hat{R}}$ instead of $D_{M \Omega}^{(J) *}(\alpha, \beta, 0) \hat{R}(\alpha, \beta, 0)$ and suppress the parametric $r$ dependence of the electronic wave functions for compactness.

\section{Action of SF angular momentum operators on basis functions}

The space-fixed electronic angular momentum operators transform under nuclear rotation as [see Eq. (3.42) of Ref. 30]

$$
\hat{R}(\alpha, \beta, 0) \underline{\hat{J}}_{\text {elec }}^{\mathrm{SF}} \hat{R}^{\dagger}(\alpha, \beta, 0)=\boldsymbol{R}^{T}(\alpha, \beta, 0) \underline{\hat{J}}_{\text {elec }}^{\mathrm{SF}}=\underline{\hat{J}}_{\text {elec }}^{\mathrm{BF}} .
$$

We have $\left[\hat{L}_{i}^{\mathrm{SF}}, \hat{S}_{j}^{\mathrm{SF}}\right]=0, \quad\left[\hat{J}_{\text {elec }, i}^{\mathrm{SF}}, D^{(J) *}(\alpha, \beta, 0)\right]=0$, and $\left[\hat{A}_{i}^{\mathrm{SF}}, \hat{A}_{j}^{\mathrm{SF}}\right]=i \epsilon_{i j k} \hat{A}_{k}^{\mathrm{SF}}$ for all SF angular momentum operators, where $\epsilon_{i j k}$ is the Levi-Civita tensor and summation over repeated indices is assumed. From these commutation relations and Eqs. (A4) and (A1) we obtain

$$
\begin{aligned}
& \hat{J}_{\text {elec }, Z}^{\mathrm{SF}} D_{M \Omega}^{(J) *} \hat{R}=D_{M \Omega}^{(J) *} \hat{R}\left[-\sin \beta \hat{J}_{\text {elec }, X}^{\mathrm{SF}}+\cos \beta \hat{J}_{\text {elec }, Z}^{\mathrm{SF}}\right] \text {, } \\
& \hat{J}_{\text {elec, },}^{\mathrm{SF}} D_{M \Omega}^{(J) *} \hat{R}=D_{M \Omega}^{(J) *} \hat{R} \mathrm{e}^{ \pm i \alpha}\left[\cos \beta \hat{J}_{\mathrm{elec}, X}^{\mathrm{SF}} \pm i \hat{J}_{\mathrm{elec}, Y}^{\mathrm{SF}}\right. \\
& \left.+\sin \beta \hat{J}_{\mathrm{elec}, Z}^{\mathrm{SF}}\right] \text {, }
\end{aligned}
$$

and similarly for $\hat{L}^{\mathrm{SF}}$ and $\underline{\hat{S}}^{\mathrm{SF}}$. Throughout this paper we define raising/lowering operators as $\hat{A}_{ \pm} \equiv \hat{A}_{X} \pm i \hat{A}_{Y}$.

The angular momentum operator $\underline{\hat{l}}^{\mathrm{SF}}$ associated with the rotation of the nuclei is the usual one-particle angular momentum operator acting on the polar angles $\alpha$ and $\beta$, as defined in Eq. (3.106) of Ref. 30. Its action on $D_{M \Omega}^{(J) *}(\alpha, \beta, 0)$ can be derived using the action of standard rigid rotor rotational operators $\hat{\mathcal{L}}(\alpha, \beta, \gamma)$ [Eq. (3.101) of Ref. 30] on a three-angle D-matrix $D_{M \Omega}^{(J) *}(\alpha, \beta, \gamma)$ and the relations

$$
\begin{aligned}
& D_{M \Omega}^{(J) *}(\alpha, \beta, 0)=D_{M \Omega}^{(J) *}(\alpha, \beta, \gamma) e^{-i \Omega \gamma}, \\
& \hat{l}_{X}^{\mathrm{SF}}=\hat{\mathcal{L}}_{X}^{\mathrm{SF}}+i \frac{\cos \alpha}{\sin \beta} \frac{\partial}{\partial \gamma},
\end{aligned}
$$

$$
\begin{aligned}
& \hat{l}_{Y}^{\mathrm{SF}}=\hat{\mathcal{L}}_{Y}^{\mathrm{SF}}+i \frac{\sin \alpha}{\sin \beta} \frac{\partial}{\partial \gamma}, \\
& \hat{l}_{Z}^{\mathrm{SF}}=\hat{\mathcal{L}}_{Z}^{\mathrm{SF}} .
\end{aligned}
$$

The result is

$$
\begin{aligned}
{\left[\hat{l}_{Z}^{\mathrm{SF}}, D_{M \Omega}^{(J) *}(\alpha, \beta, 0)\right]=} & M D_{M \Omega}^{(J) *}(\alpha, \beta, 0), \\
{\left[\hat{l}_{ \pm}^{\mathrm{SF}}, D_{M \Omega}^{(J) *}(\alpha, \beta, 0)\right]=} & c_{ \pm}(J, M) D_{M \pm 1, \Omega}^{(J) *}(\alpha, \beta, 0) \\
& -\Omega \frac{e^{ \pm i \alpha}}{\sin \beta} D_{M \Omega}^{(J) *}(\alpha, \beta, 0),
\end{aligned}
$$

where $c_{ \pm}(J, M)=[J(J+1)-M(M \pm 1)]^{1 / 2}$. The action of $\underline{\hat{l}}^{\mathrm{SF}}$ on the rotation operator $\hat{R}(\alpha, \beta, 0)$ follows from differentiation of $\hat{R}(\alpha, \beta, 0)$ with respect to $\alpha$ and $\beta$ :

$\left[\hat{l}_{Z}^{\mathrm{SF}}, \hat{R}(\alpha, \beta, 0)\right]=\hat{R}(\alpha, \beta, 0)\left[\sin \beta \hat{J}_{\mathrm{elec}, X}^{\mathrm{SF}}-\cos \beta \hat{J}_{\mathrm{elec}, Z}^{\mathrm{SF}}\right]$,

$\left[\hat{l}_{ \pm}^{\mathrm{SF}}, \hat{R}(\alpha, \beta, 0)\right]=\hat{R}(\alpha, \beta, 0) e^{ \pm i \alpha}$

$$
\times\left[-\cos \beta \hat{J}_{\mathrm{elec}, X}^{\mathrm{SF}} \mp i \hat{J}_{\mathrm{elec}, Y}^{\mathrm{SF}}+\frac{\cos ^{2} \beta}{\sin \beta} \hat{J}_{\mathrm{elec}, Z}^{\mathrm{SF}}\right] .
$$

This yields

$$
\begin{aligned}
{\left[\hat{l}_{Z}^{\mathrm{SF}}, D_{M \Omega}^{(J) *} \hat{R}\right]=} & M D_{M \Omega}^{(J) *} \hat{R}+D_{M \Omega}^{(J) *} \hat{R} \\
& \times\left[\sin \beta \hat{J}_{\mathrm{elec}, X}^{\mathrm{SF}}-\cos \beta \hat{J}_{\mathrm{elec}, Z}^{\mathrm{SF}}\right] \\
{\left[\hat{l}_{ \pm}^{\mathrm{SF}}, D_{M \Omega}^{(J) *} \hat{R}\right]=} & c_{ \pm}(J, M) D_{M \pm 1, \Omega}^{(J) *} \hat{R}+D_{M \Omega}^{(J) *} \hat{R} e^{ \pm i \alpha} \\
& \times\left[-\frac{\Omega}{\sin \beta}-\cos \beta \hat{J}_{\mathrm{elec}, X}^{\mathrm{SF}} \mp i \hat{J}_{\mathrm{elec}, Y}^{\mathrm{SF}}\right. \\
& \left.+\frac{\cos ^{2} \beta}{\sin \beta} \hat{J}_{\mathrm{elec}, Z}^{\mathrm{SF}}\right]
\end{aligned}
$$

Combining $\underline{\hat{J}}^{\mathrm{SF}}=\underline{\hat{l}}^{\mathrm{SF}}+\underline{\hat{J}}_{\text {elec }}^{\mathrm{SF}}$ yields

$$
\begin{aligned}
\hat{J}_{Z}^{\mathrm{SF}} D_{M \Omega}^{(J) *} \hat{R}|(L) \Lambda S \Sigma\rangle_{\mathrm{SF}}=M D_{M \Omega}^{(J) *} \hat{R}|(L) \Lambda S \Sigma\rangle_{\mathrm{SF}} \\
\hat{J}_{ \pm}^{\mathrm{SF}} D_{M \Omega}^{(J) *} \hat{R}|(L) \Lambda S \Sigma\rangle_{\mathrm{SF}} \\
=\left\{c_{ \pm}(J, M) D_{M \pm 1, \Omega}^{(J) *} \hat{R}+D_{M \Omega}^{(J) *} \hat{R} \frac{e^{ \pm i \alpha}}{\sin \beta}\left[\hat{J}_{\mathrm{elec}, Z}^{\mathrm{SF}}-\Omega\right]\right\} \\
\quad \times|(L) \Lambda S \Sigma\rangle_{\mathrm{SF}} .
\end{aligned}
$$

Thus the familiar standard results are only obtained when all $|(L) \Lambda S \Sigma\rangle_{\mathrm{SF}}$ are eigenfunctions of $\hat{J}_{\mathrm{elec}, Z}^{\mathrm{SF}}$ with eigenvalue $\Lambda+\Sigma$ equal to $\Omega$, so that the second term in Eq. (A18) vanishes. We chose our basis functions to have this property, and hence we have

$$
\begin{aligned}
& \hat{J}_{Z}^{\mathrm{SF}}|(L) \Lambda S \Sigma J M \Omega\rangle=M|(L) \Lambda S \Sigma J M \Omega\rangle, \\
& \left(\hat{J}^{\mathrm{SF}}\right)^{2}|(L) \Lambda S \Sigma J M \Omega\rangle=J(J+1)|(L) \Lambda S \Sigma J M \Omega\rangle .
\end{aligned}
$$




\section{Action of BF angular momentum operators on basis functions}

To derive the matrix elements for the nuclear rotational kinetic energy, we will have to look at the action of BF operators on the basis functions. We define BF operators $\underline{\hat{J}}^{\mathrm{BF}}, \underline{\underline{l}}^{\mathrm{BF}}, \underline{\hat{L}}^{\mathrm{BF}}$, and $\underline{\hat{S}}^{\mathrm{BF}}$ by

$$
\underline{\hat{A}}^{\mathrm{BF}} \equiv \mathbf{R}^{T}(\alpha, \beta, 0) \hat{A}^{\mathrm{SF}} .
$$

From the transformation property (A4) we have

$$
\underline{\hat{L}}^{\mathrm{BF}} \hat{R}(\alpha, \beta, 0)=\hat{R}(\alpha, \beta, 0) \underline{\hat{L}}^{\mathrm{SF}}
$$

and

$$
\underline{\hat{S}}^{\mathrm{BF}} \hat{R}(\alpha, \beta, 0)=\hat{R}(\alpha, \beta, 0) \underline{\hat{S}}^{\mathrm{SF}},
$$

and, using $\left[\hat{L}_{i}^{\mathrm{BF}}, D_{M \Omega}^{(J) *}\right]=\left[\hat{S}_{i}^{\mathrm{BF}}, D_{M \Omega}^{(J) *}\right]=0$ we find

$$
\begin{aligned}
& \hat{L}_{Z}^{\mathrm{BF}}|(L) \Lambda S \Sigma J M \Omega\rangle=\Lambda|(L) \Lambda S \Sigma J M \Omega\rangle, \\
& \hat{S}_{Z}^{\mathrm{BF}}|(L) \Lambda S \Sigma J M \Omega\rangle=\Sigma|(L) \Lambda S \Sigma J M \Omega\rangle, \\
& \hat{S}_{ \pm}^{\mathrm{BF}}|(L) \Lambda S \Sigma J M \Omega\rangle=c_{ \pm}(S, \Sigma)|(L) \Lambda S \Sigma \pm 1 J M \Omega\rangle .
\end{aligned}
$$

Even though the electronic states are not eigenfunctions of $\left(\hat{L}^{\mathrm{BF}}\right)^{2}$ we still have $\hat{L}_{ \pm}^{\mathrm{BF}}|(L) \Lambda S \Sigma J M \Omega\rangle=D_{M \Omega}^{(J) *} \hat{R} \hat{L}_{ \pm}^{\mathrm{SF}}$ $|(L) \Lambda S \Sigma\rangle_{\mathrm{SF}}$. Substituting the expressions for $\underline{\hat{l}}^{\mathrm{SF}}$ into Eq. (A21) gives $\hat{l}_{Z}^{\mathrm{BF}}=0$ and

$$
\hat{l}_{ \pm}^{\mathrm{BF}}=\frac{i}{\sin \beta} \frac{\partial}{\partial \alpha} \pm \frac{\partial}{\partial \beta} .
$$

Using the relations between $\hat{l}_{ \pm}^{\mathrm{BF}}$ and the rigid rotor BF operators [Eq. (3.122) in Ref. 30], and Eq. (A7) we find

$$
\begin{gathered}
\hat{l}_{ \pm}^{\mathrm{BF}} D_{M \Omega}^{(J) *}(\alpha, \beta, 0)=c_{\mp}(J, \Omega) D_{M, \Omega \mp 1}^{(J) *}(\alpha, \beta, 0) \\
-\Omega \cot \beta D_{M \Omega}^{(J) *}(\alpha, \beta, 0), \\
\hat{l}_{ \pm}^{\mathrm{BF}} \hat{R}(\alpha, \beta, 0)=\hat{R}(\alpha, \beta, 0)\left[-\hat{J}_{\text {elec, } \pm}^{\mathrm{SF}}+\cot \beta \hat{J}_{\text {elec }, Z}^{\mathrm{SF}}\right],
\end{gathered}
$$

yielding

$$
\begin{aligned}
\hat{l}_{ \pm}^{\mathrm{BF}} D_{M \Omega}^{(J)} \hat{R}= & c_{\mp}(J, \Omega) D_{M, \Omega \mp 1}^{(J) *} \hat{R} \\
& +D_{M \Omega}^{(J) *} \hat{R}\left\{-\hat{J}_{\text {elec, } \pm}^{\mathrm{SF}}+\cot \beta\left[\hat{J}_{\text {elec }, Z}^{\mathrm{SF}}-\Omega\right]\right\} .
\end{aligned}
$$

We finally obtain

$\hat{J}_{Z}^{\mathrm{BF}}|(L) \Lambda S \Sigma J M \Omega\rangle=\Omega|(L) \Lambda S \Sigma J M \Omega\rangle$,

$\hat{J}_{ \pm}^{\mathrm{BF}}|(L) \Lambda S \Sigma J M \Omega\rangle=c_{\mp}(J, \Omega)|(L) \Lambda S \Sigma J M \Omega \mp 1\rangle$.

\section{Rotational Hamiltonian}

The Hamiltonian for the nuclear rotational kinetic energy is

$$
\begin{aligned}
\hat{H}_{\mathrm{rot}}=\frac{1}{2 \mu r^{2}}\left(\hat{l}^{\mathrm{SF}}\right)^{2}= & \frac{1}{2 \mu r^{2}}\left[\left(\hat{J}^{\mathrm{SF}}\right)^{2}+\left(\hat{L}^{\mathrm{SF}}\right)^{2}+\left(\hat{S}^{\mathrm{SF}}\right)^{2}\right. \\
& \left.-2 \underline{\hat{L}}^{\mathrm{SF}} \cdot \underline{\hat{J}}^{\mathrm{SF}}-2 \underline{\hat{S}}^{\mathrm{SF}} \cdot \underline{\hat{J}}^{\mathrm{SF}}+2 \underline{\hat{L}}^{\mathrm{SF}} \cdot \underline{\hat{S}}^{\mathrm{SF}}\right] .
\end{aligned}
$$

Using the orthogonality of $\mathbf{R}(\alpha, \beta, 0)$ we can derive that $\left[\hat{L}_{i}^{\mathrm{BF}}, \hat{L}_{j}^{\mathrm{BF}}\right]=i \epsilon_{\mathrm{ijk}} \hat{L}_{k}^{\mathrm{BF}}$ and $\left[\hat{S}_{i}^{\mathrm{BF}}, \hat{S}_{j}^{\mathrm{BF}}\right]=i \epsilon_{i j k} \hat{S}_{k}^{\mathrm{BF}}$. Furthermore $\left[R_{i j}(\alpha, \beta, 0), \hat{L}_{k}^{\mathrm{BF}}\right]=\left[R_{i j}(\alpha, \beta, 0), \hat{S}_{k}^{\mathrm{BF}}\right]=0$, and thus

$\underline{\hat{A}}^{\mathrm{SF}} \cdot \underline{\hat{B}}^{\mathrm{SF}}=\underline{\hat{A}}^{\mathrm{BF}} \cdot \underline{\hat{B}}^{\mathrm{BF}}=\frac{1}{2}\left(\hat{A}_{+}^{\mathrm{BF}} \hat{B}_{-}^{\mathrm{BF}}+\hat{A}_{-}^{\mathrm{BF}} \hat{B}_{+}^{\mathrm{BF}}\right)+\hat{A}_{z}^{\mathrm{BF}} \hat{B}_{z}^{\mathrm{BF}}$

for the inner products $\left(\hat{S}^{\mathrm{SF}}\right)^{2}, \underline{\hat{L}}^{\mathrm{SF}} \cdot \underline{\hat{J}}^{\mathrm{SF}}, \underline{\hat{S}}^{\mathrm{SF}} \cdot \underline{\hat{J}}^{\mathrm{SF}}$, and $\underline{\hat{L}}^{\mathrm{SF}}$ $\cdot \underline{\hat{S}}^{\mathrm{SF}}$. Since $\hat{l}_{Z}^{\mathrm{BF}}=0$ we have $\hat{J}_{Z}^{\mathrm{BF}}=\hat{L}_{Z}^{\mathrm{BF}}+\hat{S}_{Z}^{\mathrm{BF}}$, and we can rewrite the rotational Hamiltonian as Eq. (4), where we dropped the $\mathrm{BF}$ label on the BF operators, and used small $z$, and superscript \pm for $\mathrm{BF}$ components, in accordance to the notation of Lefebvre-Brion. ${ }^{21}$

\section{Parity label}

The space fixed inversion operator $\hat{i}$ acts on both nuclear and electronic coordinates. It commutes with all SF angular momentum operators, it has the properties $\hat{i}^{\dagger} \hat{i}=1$ and $\hat{i}$ $=\hat{i}^{\dagger}$, and its action on the polar angles $\alpha$ and $\beta$ is given by $\hat{i} \alpha \hat{i}^{\dagger}=\alpha+\pi$ and $\hat{i} \beta \hat{i}^{\dagger}=\pi-\beta$. Its action on the Wigner D-matrix is given by

$$
\begin{aligned}
\hat{i} D_{M \Omega}^{(J) *}(\alpha, \beta, 0) \hat{i}^{\dagger} & =\exp [i M(\alpha+\pi)] d_{M \Omega}^{J}(\pi-\beta) \\
& =\exp (-i \pi J) D_{M,-\Omega}^{(J) *}(\alpha, \beta, 0),
\end{aligned}
$$

where we used Eqs. (3.67) and (3.75) of Ref. 31 in the second step. To derive the action of $\hat{i}$ on the rotation operator we first observe that from Eq. (A4) we have

$$
\hat{R}_{Z}(\pi) \hat{J}_{Y} \hat{R}_{Z}^{\dagger}(\pi)=-\hat{J}_{Y}
$$

and hence

$$
\hat{R}_{Z}(\pi) \hat{R}_{Y}(-\beta) \hat{R}_{Z}^{\dagger}(\pi)=\hat{R}_{Y}(\beta) .
$$

Using this relation one can show that

$$
\begin{aligned}
\hat{i} \hat{R}(\alpha, \beta, 0) \hat{i}^{\dagger} & =\hat{R}_{Z}(\alpha) \hat{R}_{Z}(\pi) \hat{R}_{Y}(-\beta) \hat{R}_{Y}(\pi) \\
& =\hat{R}(\alpha, \beta, 0) \hat{R}_{Y}(\pi) \hat{R}_{Z}(-\pi) .
\end{aligned}
$$

With the use of $\hat{R}_{Z}(-\pi)|(L) \Lambda S \Sigma\rangle_{\mathrm{SF}}=\exp [i \pi(\Lambda+\Sigma)]$ $|(L) \Lambda S \Sigma\rangle_{\mathrm{SF}}$ and $\left[\hat{R}_{Z}(-\pi), \hat{i}\right]=0$ we derive

$\hat{i}|(L) \Lambda S \Sigma J M \Omega\rangle=\exp [-i \pi(J-\Omega)] D_{M,-\Omega}^{(J) *}(\alpha, \beta, 0)$

$$
\times \hat{R}(\alpha, \beta, 0) \hat{R}_{Y}(\pi) \hat{i}|(L) \Lambda S \Sigma\rangle_{\mathrm{SF}} .
$$

Thus, to work out this expression we only have to apply $\hat{R}_{Y}(\pi) \hat{i}$ to $|(L) \Lambda S \Sigma\rangle_{\mathrm{SF}}$, which is the electronic wave function in the space-fixed frame as obtained from the ab initio calculation. Since $\left[\hat{i}, \hat{L}_{Z}\right]=\left[\hat{i}, \hat{S}_{Z}\right]=0$, we find that $\hat{i}$ does not change the value of $\Omega$ in the ket $|(L) \Lambda S \Sigma\rangle_{\mathrm{SF}}$. SF inversion does not act on spin coordinates, i.e., $\hat{i}|S \Sigma\rangle_{\mathrm{SF}}$ $=|S \Sigma\rangle_{\mathrm{SF}}$, and kets can be rotated with a Wigner D-matrix, so $\quad \hat{R}_{Y}(\pi) \hat{i}|S \Sigma\rangle_{\mathrm{SF}}=\Sigma_{\Sigma^{\prime}}\left|S \Sigma^{\prime}\right\rangle_{\mathrm{SF}} D_{\Sigma^{\prime} \Sigma}^{(S)}(0, \pi, 0)=(-1)^{S-\Sigma}$ $|S-\Sigma\rangle_{\mathrm{SF}}$. For the spatial part we have $\hat{R}_{Y}(\pi) \hat{i}|(L) \Lambda\rangle_{\mathrm{SF}}$ $=\hat{\sigma}_{v}(X Z)|(L) \Lambda\rangle_{\mathrm{SF}}$, where $\hat{\sigma}_{v}(X Z)$ denotes reflection in the $\mathrm{SF} X Z$ plane. For one-electron orbitals described by a spherical harmonic we have $\hat{\sigma}_{v}(X Z)|l \lambda\rangle=(-1)^{\lambda}|l-\lambda\rangle$, and for a many-electron ket $\left|\left(l_{i}\right) L \Lambda\right\rangle$ consisting of Clebsch-Gordan coupled one-electron kets we find $\hat{\sigma}_{v}(X Z)\left|\left(l_{i}\right) L \Lambda\right\rangle$ 
$=(-1)^{\sum_{i} l_{i}+L+\Lambda}\left|\left(l_{i}\right) L-\Lambda\right\rangle$. For a diatomic molecule $L$ is not a good quantum number, but a state that is asymptotically $\Sigma^{ \pm}$, will be $\Sigma^{ \pm}$for finite $R$ also. All our states are asymptotically $\mathrm{O}\left({ }^{3} P\right)+\mathrm{O}\left({ }^{3} P\right)$, with four $p$ and four $s$ electrons per atom. Thus $\hat{\sigma}_{v}(X Z)|(L) \Lambda\rangle_{\mathrm{SF}}=(-1)^{L+\Lambda}|(L)-\Lambda\rangle_{\mathrm{SF}}, \quad$ yielding $\hat{R}_{Y}(\pi) \hat{i}|(L) \Lambda S \Sigma\rangle_{\mathrm{SF}}=(-1)^{S-\Sigma+L+\Lambda}|(L)-\Lambda S-\Sigma\rangle_{\mathrm{SF}}$, and finally

$\hat{i}|(L) \Lambda S \Sigma J M \Omega\rangle=(-1)^{J+L-S}|(L)-\Lambda S-\Sigma J M-\Omega\rangle$.

For our ungerade states $(-1)^{L-S}$ is odd. ${ }^{14}$

The ${ }^{16} \mathrm{O}$ isotope has nuclear spin $I=0$. Hence, from the Pauli principle for bosons, it follows that the spatial part of the nuclear wave function must be symmetric under space fixed inversion. Thus, for the ungerade electronic states of ${ }^{16} \mathrm{O}{ }^{16} \mathrm{O}$ only odd parity wave functions are allowed.

\section{ACKNOWLEDGMENTS}

The authors thank Ad van der Avoird and Paul E. S. Wormer for useful discussions and for carefully reading the manuscript. This research has been financially supported by the Council for Chemical Sciences of the Netherlands Organization for Scientific Research (CW-NWO).

${ }^{1}$ G. Herzberg, Naturwissenschaften 20, 577 (1932).

${ }^{2}$ G. Herzberg, Can. J. Phys. 30, 185 (1952).

${ }^{3}$ G. Herzberg, Can. J. Phys. 31, 657 (1953).

${ }^{4}$ D. A. Ramsay, Can. J. Phys. 64, 717 (1986).

${ }^{5}$ P. M. Borrell, P. Borrell, and D. A. Ramsay, Can. J. Phys. 64, 721 (1986).

${ }^{6}$ B. Coquart and D. A. Ramsay, Can. J. Phys. 64, 726 (1986).

${ }^{7}$ T. G. Slanger and P. C. Cosby, J. Phys. Chem. 92, 267 (1988)

${ }^{8}$ T. G. Slanger, D. L. Huestis, P. C. Cosby, H. Naus, and G. Meijer, J. Chem. Phys. 105, 9393 (1996).

${ }^{9}$ K. Yoshino, J. R. Esmond, J. E. Murray, W. H. Parkinson, A. P. Thorne, R. C. M. Learner, and G. Cox, J. Chem. Phys. 103, 1243 (1995).
${ }^{10}$ K. Yoshino, J. R. Esmond, W. H. Parkinson, A. P. Thorne, R. C. M. Learner, and G. Cox, J. Chem. Phys. 111, 2960 (1999).

${ }^{11}$ K. Yoshino, J. R. Esmond, W. H. Parkinson, A. P. Thorne, R. C. M. Learner, G. Cox, and A. S.-C. Cheung, J. Chem. Phys. 112, 9791 (2000).

${ }^{12}$ M. C. G. N. van Vroonhoven and G. C. Groenenboom, J. Chem. Phys. 116, 1954 (2002).

${ }^{13}$ M. C. G. N. van Vroonhoven and G. C. Groenenboom, J. Chem. Phys. 116, 1965 (2002).

${ }^{14}$ B. Zygelman, A. Dalgarno, and R. D. Sharma, Phys. Rev. A 49, 2587 (1994).

${ }^{15}$ B. Zygelman, A. Dalgarno, and R. D. Sharma, Phys. Rev. A 50, 3920 (1994).

${ }^{16}$ D. R. Bates, Proc. Phys. Soc. London, Sect. B 64, 805 (1951).

${ }^{17}$ R. P. Saxon and B. Liu, J. Chem. Phys. 67, 5432 (1977).

${ }^{18}$ H. Partridge, C. W. Bauschlicher, Jr., S. R. Langhoff, and P. R. Taylor, J. Chem. Phys. 95, 8292 (1991).

${ }^{19}$ A. Jenouvrier, M.-F. Mérienne, B. Coquart, M. Carleer, S. Fally, A. C. Vandaele, C. Hermans, and R. Colin, J. Mol. Spectrosc. 198, 136 (1999).

${ }^{20}$ J. T. Hougen, J. Chem. Phys. 36, 519 (1962).

${ }^{21}$ H. Lefebvre-Brion and R. W. Field, Perturbations in the Spectra of Diatomic Molecules (Academic, New York, 1986).

${ }^{22}$ G. C. Groenenboom and D. T. Colbert, J. Chem. Phys. 99, 9681 (1993), note the mistake in the signs between the two terms in the first and second part of Eq. (48); see also D. T. Colbert and W. H. Miller, ibid. 96, 1982 (1992).

${ }^{23}$ M. Mizushima, The Theory of Rotating Diatomic Molecules (Wiley, New York, 1975)

${ }^{24}$ J. T. Hougen, "The calculation of rotational energy levels and rotational line intensities in diatomic molecules," NBS Monograph 115, National Bureau of Standards, 1970, available online at http://physics.nist.gov/ Pubs/Mono115/.

${ }^{25}$ J. H. van Vleck, Rev. Mod. Phys. 23, 213 (1951).

${ }^{26}$ J. K. G. Watson, Mol. Phys. 19, 465 (1970).

${ }^{27}$ G. Brocks, A. van der Avoird, B. T. Sutcliffe, and J. Tennyson, Mol. Phys. 50, 1025 (1983).

${ }^{28}$ A. van der Avoird, P. E. S. Wormer, and R. Moszynski, Chem. Rev. 94, 1931 (1994).

${ }^{29}$ M. Larsson, Phys. Scr. 23, 835 (1981).

${ }^{30}$ L. C. Biedenharn and J. D. Louck, Angular Momentum in Quantum Physics, Vol. 8 of Encyclopedia of Mathematics (Addison-Wesley, Reading, 1981).

${ }^{31}$ R. N. Zare, Angular Momentum (Wiley, New York, 1988). 